\title{
Peron e il miraggio del Blocco latino Di come la guerra fredda allargò l'Atlantico Sud*
}

\author{
Loris Zanatta
}

Università di Bologna

Basada en los archivos diplomáticos de Argentina, Brasil, Chile, España, Italia, Perú y Portugal, esta investigación analiza el intento del gobierno de Perón de formar un bloque de países latinos capaz de reunir bajo su hegemonía y con la legitimación de la Santa Sede las naciones católicas de Europa y de América. Conocido como la "tercera posición", ese bloque fracasó a medida que la guerra fría imponía sus reglas y creaba las condiciones de una inédita alianza entre la mayoría de los países católicos y el Vaticano con Estados Unidos. Como consecuencia la política peronista se alejó de su origen confesional para evolucionar hacia una ideología cada vez más tercermundista. Al mismo tiempo el aborto del bloque latino demostró que el sistema bipolar destruía la noción de civilización latina diluyéndola en la más amplia de Occidente cristiano.

Palabras Clave: Peronismo, América Latina y Guerra Fría, Relaciones Internacionales, Naciones católicas, Hispanidad y Latinidad, Santa Sede.

This article is based upon a multi-archival research in the diplomatic archives of seven different countries: Argentina, Brazil, Chile, Spain, Italy, Perù and Portugal. It analyzes the attempt of the Peròn government to form a bloc of Latin countries, gathering under its hegemony, and with the support of the Vatican, the catholic nations of Europe and America. Known as the "third position", the idea of creating such a bloc failed once the Cold War imposed its rules, laying the ground for an odd alliance between most catholic countries, the Vatican and the United States. As a consequence, Peròn's policy abandoned its religious matrix and drifted progressively toward third-worldist horizons. At the same time, the abortion of the Latin bloc showed the capability of the bipolar system to erode the notion of a Latin civilization, diluting it into the broadest one of the Christian West.

Keywords: Peronism; Latin America and Cold War, International Relations, Catholic nations, Latinity and Hispanidad, Holy See.

La guerra fredda avanzava implacabile e le rovine di quella appena conclusa fumavano ancora, quando Juan Domingo Peron annunciò al mondo la sua Terza Posizione: né con gli Stati Uniti né con l'Unione Sovietica,

* Este artículo es fruto de una investigación financiada por el Ministero dell’Università e della Ricerca Scientifica de Italia sobre "Il posto dell'Europa nella storia americana". Agradezco mucho al Ministerio y a los colegas de las Universidades del Piamonte Oriental, Bologna y Florencia que formaron parte del mismo proyecto. 
né capitalismo né comunismo, né individualismo né collettivismo. Giovane e tronfia, rigogliosa e velleitaria, orgogliosa e sbruffona, la sua Argentina usciva dal coro e sfidava il nuovo ordine mondiale. Vien da sorriderne, col senno di poi. Ma in quel mondo storpiato, tutto fame e miseria, lutto e paura, l'Argentina era una Mecca. O tale pareva. Crediti e terra, pace e cibo; carne e grano in abbondanza, da sfamarci il mondo, e spazi immensi e vuoti dove accogliere diseredati in quantità. Peron ne era lo specchio. Sottratto a furor di popolo dalle patrie galere, aveva mondato la macchia fascista dal suo pedigree trionfando in elezioni pulite; isolato e vituperato dagli Stati Uniti, li aveva vinti e umiliati. Tutto salute e sorrisi, scaltrezza e magniloquenza, era l'immagine del successo e della baldanzosa arroganza di un paese deputato ad un grande destino. Chi ne dubitava?

L'Argentina di Peron, insomma, contava. Lo dimostrano gli sforzi statunitensi per placarne l'ostilità e blandirne l'ego sperando di farne un solido pilastro della comunità panamericana; lo confermano le reiterate preghiere vaticane perché elargisse cibo e permessi migratori per placare gli incendi nell'Europa assediata dal comunismo. Tutti e ovunque la trattavano con tatto e timore per averne grano in più a prezzi buoni: la Spagna di Franco vi si aggrappò per galleggiare tra fame e isolamento; i vicini l'ammiravano e temevano, la corteggiavano perché non lesinasse la chiave della dispensa, la detestavano per le sue smodate ambizioni. Non che pesasse quanto Peron credeva, ma certo quell' Argentina aveva voce in capitolo.

Sulla Terza Posizione s'è detto e scritto molto ${ }^{1}$. Di certo non fu intesa e applicata allo stesso modo dalle diverse "famiglie" del peronismo. Molti militari vi colsero il sale ideologico della vocazione argentina al primato geopolitico in America Latina; la potente CGT, il sindacato peronista, spalleggiata dall'orchestra di radio e giornali diretta da Eva Peron, l'usò per esportare il peronismo tra gli operai dell'intero continente. Gli industriali e avventurieri fioriti o ingrassati all'ombra di Peron ne celebrarono il sogno d'indipendenza economica, foriero di protezione e di trattati convenienti per assicurarsi materie prime e sbocchi commerciali. Alla Chiesa, infine, e ai cattolici saltati con gioia e compunzione sul carro peronista, la Terza Posizione parve la sacrosanta rivendicazione della civiltà cattolica in un

1 Véase. Escudé, C.: Gran Bretaña, Estados Unidos y la declinación argentina, 1942-1949, Editorial de Belgrano, Buenos Aires, 1983; Rapoport, M., Spiguel, C.: Estados Unidos y el peronismo: la política norteamericana en la Argentina, 1949-1955, Grupo Editor Latinoamericano, Buenos Aires, 1994; Paradiso, J.: Vicisitudes de una política exterior independiente, en Torre, J. C. (ed.): "Los años peronistas (1943-1955)", Nueva Historia Argentina, t. VIII, págs. 523-572. 
mondo stretto tra una potenza atea e una protestante e dell'aspirazione argentina a guidare il rinascimento cattolico in America Latina.

Al tempo stesso, sotto le sgargianti fogge della variopinta galassia peronista, ricoperta di grida rivoluzionarie, la Terza Posizione rinverdiva gli antichi fasti dell' eccezionalismo argentino; rilanciava, insomma, il mito vecchio e solido di una nazione votata al primato, lanciata nel futuro, crogiolo di speranza, progresso e gioventù. Lo nutrivano la sua popolazione colta e bianca, il suo territorio ricco e sterminato, l'epopea della frontiera e della nuova civiltà costruita laddove un tempo regnavano deserto e barbarie. E soprattutto le sue robuste radici europee, così ricche in sangue, soldi, lettere e preghiere da farne un pezzo d'Europa in fondo all'America india, nera e meticcia; da alimentarne l'alterigia e stabilirne la missione: dapprima di faro in un continente afflitto da miseria e ignoranza e poi, man mano che i demoni si impossessavano dell'Europa nelle trincee delle sue folli guerre, di erede e rigeneratrice della sua civiltà sul suolo americano. Come tale, com'è vero per due galli in un pollaio, l'eccezionalismo argentino era votato alla collisione con quello statunitense, così uguale e così contrario. Ebbene, la Terza Posizione lo confermava, col suo perentorio rifiuto di far salire l'Argentina con armi, bagagli e mansuetudine sul treno del panamericanismo pilotato dal presidente Truman lungo le praterie di un continente dove, finita la guerra, ogni significativa traccia d'influenza europea pareva destinata a diventare un remoto ricordo.

Detto ciò, la Terza Posizione fu il modo, senz'altro evocativo all'alba del mondo bipolare, in cui il presidente Peron ribattezzò un'idea già cara al colonnello Peron quando ancora regnava il regime militare nato il 4 giugno 1943. Di fatto era l'organica proiezione nell'arena internazionale del suo mondo ideale, comune a molti suoi commilitoni e connazionali; un mondo tutt'altro che inedito nel panorama dell'epoca, ma ch'egli chiamò pomposamente justicialismo. Il quale, ridotto all'osso, era una sorta di terza via filosofica e politica di chiara matrice cattolica e antiliberale; una via corporativa, fondata su una concezione sociale organicistica, ugualmente distante dalle democrazie liberali e dal comunismo sovietico ${ }^{2}$. La Terza Posizione ne era il riflesso, la filiazione: lo era stata durante la guerra, quando il governo militare era giunto sull'orlo del suicidio pur di restare neutrale nonostante le tremende pressioni statunitensi; e lo fu in seguito, quando

2 Véase Zanatta, L.: "Filosofia Politica", Il populismo in America Latina. Il volto moderno di

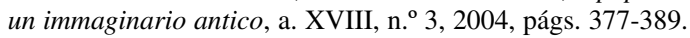


Peron ne fece il vessillo dell'insofferenza argentina per le ferree ed arbitrarie regole della guerra fredda. I militari l'avevano invocata sognando di portare l'Argentina sul binario morto dei "fascismi cattolici" che correva fiero e puro tra la civiltà liberale anglosassone e quella comunista del "barbaro" Oriente; finita la guerra, Peron la ribadì per tenere alta la bandiera dell'eccezionalismo argentino, sottrarsi alla scelta di campo e schivare l'ombra statunitense che si proiettava sempre più lunga sui destini dell'America Latina. Se per un verso, dunque, la Terza Posizione era figlia della terza via fascista, per un altro prefigurava le multiformi varianti di neutralismo destinate a sorgere tra le pieghe della guerra fredda.

Fin qui nulla, o quasi, di nuovo. Sennonché la Terza Posizione fu anche uno straordinario termometro dell'impatto prodotto dalla guerra mondiale e dalla guerra fredda sui rapporti tra Europa e America Latina. In parte, infatti, essa fu l'ultimo, vago ma ambizioso progetto di fondare, contro venti e maree, la storia e l'economia, la forza e la prudenza, una comunità di nazioni a cavallo dell' Atlantico, tra Europa e Sud America. Un progetto fumoso, ma inseguito con tenacia e senza badare a spese; quasi un sogno, o un ideale: quello di aggregare, sotto la guida politica argentina e quella morale della Santa Sede, la comunità immaginaria delle nazioni cattoliche e latine d'America e d'Europa. Come se alla "nuova Argentina", modello di cristianità e giustizia sociale, trionfante contro il miraggio comunista e il capitalismo selvaggio, spettasse la missione di resuscitare e riunire l'Europa cattolica assediata dal comunismo e l'America Latina soggiogata dall'imperialismo; fino a formare un blocco indipendente dai Blocchi.

Per un po', finché l'alleanza di guerra tra Stati Uniti e Unione Sovietica non si trasformò in aperto conflitto, l'idea peronista di "terza via" non parve così campata per aria. Man mano, però, che la trama bipolare del nuovo mondo divenne più nitida e impose regole e scelte, anche la vecchia nozione di "civiltà" latina cara a Peron e alla Terza Posizione cedette il passo a una categoria nuova, più ampia e comprensiva nella sua diafana semplicità: l'Occidente cristiano. Il sogno di Peron cominciò allora a perdere pezzi, uno dopo l'altro; dapprima, e soprattutto, in Europa, tutti fatalmente attratti dalla portentosa calamita della guerra fredda, che per paura o ammirazione, riconoscenza, debito o convinzione, li attrasse nell'orbita di Washington. Finché lo stesso Peron, chiuso in quella strada senz'uscita, cercò di aprirsi un dignitoso varco verso la capitale dell'Impero, salvo trovarlo sbarrato; non dall' astio statunitense verso il figliol prodigo che timi- 
damente si univa al gregge panamericano, ma dal pedaggio reclamato dalla Terza Posizione: come accantonarla, infatti, senza tradire se stesso e il suo popolo, plasmato dalle suadenti sirene del justicialismo?

La mesta parabola della Terza Posizione, in sintesi, misura quanto la guerra mondiale e la guerra fredda allargarono l'Atlantico Sud mentre restringevano l'Atlantico Nord; quanto accelerarono il secolare distacco latinoamericano dalle radici europee, perfino nelle sue propaggini più estreme e resistenti come l'Argentina, mentre avvicinavano l'Europa agli Stati Uniti. Certo, la Terza Posizione sollevò vaste speranze e passioni in America Latina e non cessa di riaffiorare in forme e luoghi ogni volta diversi. Ma più che rianimare un antico e perduto legame col Vecchio Mondo è da allora un mero abito del vasto e poliedrico nazionalismo latinoamericano. Come tale, anch'essa testimonia della progressiva, inesorabile americanizzazione dell'America Latina.

\section{Dalle stalle alle stelle. L'elezione di Peron e l'alba radiosa della Terza Posizione}

Se l'era vista brutta, Peron, nel 1945, quando la sua folgorante carriera politica parve chiudersi bruscamente. Certo, man mano che le truppe alleate avanzavano e l'emisfero americano si raccoglieva per amore o per forza intorno agli Stati Uniti, anch'egli aveva mollato zavorra, inimicandosi i più stolidi nazionalisti. Così alla fine si era deciso a dichiarare guerra all'Asse un istante prima che capitolasse; quanto bastava perché Washington sfidasse l'alleato sovietico, deciso a punire quel militare in odore di fascismo, prendendo l'Argentina alle Nazioni Unite. Non per questo, va da sé, alla Casa Bianca intendevano rassegnarsi alla convivenza con l'odiato colonnello; ne volevano la testa, e tra il 1943 e il 1946 fecero di tutto per averla, finché, messi alle strette, i militari argentini indissero elezioni. L'ira di Washington non conobbe limiti. Il governo militare boliviano scimmiottava quello argentino? C'eran voluti sei mesi e la purga dei ministri sospetti di connivenza con Buenos Aires perché gli Stati Uniti lo riconoscessero ${ }^{3}$; il fascismo era caduto e l'Italia liberata pendeva da Washington? Che rompesse con l'Argentina ${ }^{4}$. Il governo peruviano voleva

3 Véase Zanatta, L.: "Desarrollo Economico", Bolivia, Perón y la Guerra Fría, 1943-1954. Auge y Declinación de la Tercera Posición, n. ${ }^{\circ}$ 177, abril-junio, 2005, págs. 25-53. 
scambiare petrolio col grano argentino? Gli Stati Uniti mettevano i bastoni tra le ruote 5 . Il Brasile s'era impegnato con Buenos Aires a fornire i pneumatici perché i suoi camion trasportassero il grano appena mietuto? Niente da fare, fecero sapere al Dipartimento di Stato: il "virus" di Braden, la bestia nera di Peron, "sta producendo i suoi effetti", confidò Getulio Vargas $^{6}$. E così via. Peron doveva sparire: fatti scegliere, cioè votare, gli argentini avrebbero senz'altro scelto l'unità panamericana, l'amicizia con Washington, il matrimonio con la democrazia liberale. Peron, dunque, se la vide davvero brutta e la sua incarcerazione, nell'ottobre 1945, parve segnarne il declino e quello della sua terza via.

Ma le cose andarono altrimenti e la ciambella cucinata da Braden con tanta dovizia uscì senza buco. La folla, infatti, volle il ritorno di Peron e i militari lo accolsero con sollievo pur di evitare una vergognosa ritirata dal potere. Non per questo gli Stati Uniti si rassegnarono e col Libro Azul pensarono di inchiodare Peron alla croce della sua amicizia con l'Asse; ma ne uscì un goffo boomerang, assai ghiotto per il gaudente colonnello, il quale, incitando alla scelta tra lui e lo yankee portò a casa uno strepitoso successo elettorale, conficcandosi come un osso di traverso nella gola dello zio Sam, dove rimase un intero decennio. Peron, dunque, era tornato; anzi, non se n'era mai andato. Piacesse o meno, non era più il brutto anatroccolo figliato da una dittatura, ma un presidente costituzionale con tutti i sacri crismi. Forte più che mai, colmo d'astio ed entusiasmo, le piazze, i silos e le casse piene in quel mondo di stenti, Peron non tardò un istante a riprendere i fili della rivoluzione, precisare i contorni del justicialismo, ricamare la trama della Terza Posizione; di qua e di là dall'Atlantico. Armi in mano ne aveva; specie il grano. La guerra aveva devastato campi e granai; come stupirsi che code di questuanti si assiepassero alle porte argentine? E non era solo fame: l'incubo della rivolta comunista, incubata nelle code per il pane razionato, popolava i sonni dei governi d'Europa e d'America, di Truman e Pio XII ${ }^{7}$. Già nel 1945, e di nuovo in seguito, non a caso, ora in pubblico ora in privato, il Pontefice s'appellò alla generosità argentina, ben sapendo

4 Archivo del Ministero degli Esteri italiano, AMAEI, Memorandum for the Allied Control Commission, 15 de agosto de 1944.

5 Archivo del Ministerio de Relaciones Exteriores de Chile, AMREC, Lima a Santiago, 24 de noviembre de 1945.

6 Véase Archivo del Ministerio de Relaciones Exteriores de la Argentina, AMREA, Río de Janeiro a Buenos Aires, 18 de octubre de 1945.

7 Véase Di Nolfo, E.: Vaticano e Stati Uniti. 1939-1952. Dalle carte di Myron C. Taylor, FrancoAngeli, Milano, 1978. 
di potervi contare, dato il fervore con cui Peron s'ergeva ad apostolo di Cristo e bastione della civiltà cattolica ${ }^{8}$.

Difatti non si può dire che il governo argentino si tirasse indietro, benché tanta premura generasse qualche sospetto. Non sarà, qualcuno si chiese, che gli aiuti argentini celavano un disegno politico? Che fungessero da grimaldello della Terza Posizione? Non erano, si badi, le solite, velenose voci di corridoio delle cancellerie. Interessata o meno, fu la grande stampa anglosassone a lanciare il grido d'allarme. All'inizio del 1946, per dire, Times e Daily Telegraph non ci andarono per il sottile: l'Argentina, scrissero, privilegiava Spagna e Portogallo perché aveva in mente un Blocco latino9. Calunnie? Buenos Aires, va da sé, smentì, ma le accuse non scomparvero. Anzi, crebbero col tempo. D'altra parte non erano infondate e farisaiche o meno pesavano come macigni: suonava cinico sfamare alcuni più d'altri; visti i trascorsi di Peron, poi, c'era chi scorgeva nei suoi piani la rinascita di un'internazionale fascista.

In barba alle smentite il governo argentino aveva davvero privilegiato i paesi iberici e i loro devoti dittatori. I diplomatici portoghesi in Argentina, commossi dalle tenere ed ammirate parole di Peron per Salazar, sapevano di avere avuto ciò che ad altri era negato. Intimoriti dai toni della stampa inglese ne parlarono coi colleghi spagnoli, decisi a non farvi caso; in realtà, confidò sornione l'Ambasciatore di Franco, la Spagna aveva avuto ancor più grano di quanto fosse noto; e l'importante, aggiunse, era proprio il valore politico di tanta premura argentina ${ }^{10}$. L'uso politico del grano al servizio della Terza Posizione, benché destinato a divenire un'arma spuntata, fu lungo e persistente ${ }^{11}$. Verso l'Europa, come s'è visto, e verso i vicini americani, i più piccini e ricattabili ${ }^{12}$, ma anche $i$ più grandi: il Brasile, per esempio, drammaticamente a corto di grano e rimasto ormai senza pane. La situazione sociale - scriveva l'Ambasciatore argentino a Rio nel febbraio

8 AMREA, Santa Sede a Buenos Aires, 5 de diciembre de 1945; L'Osservatore Romano, 5 de abril de 1946; Arquivo do Ministério dos Negòcios Estrangeiros do Portugal, AMNEP, Roma a Lisbona, 5 de abril de 1946. Sul primo Peron e la Chiesa, véase Caimari, L.: Perón y la Iglesia católica, Ariel, Buenos Aires, 1994.

9 AMNEP, Buenos Aires a Lisbona, 23 de marzo de 1946.

10 AMNEP, Buenos Aires a Lisbona, 13 y 24 de marzo de 1946.

11 Dorn, G.J.: "Perón's Gambit: The United States and the Argentine Challenge to the InterAmerican Order, 19461948”, Diplomatic History, v. 26, n. ${ }^{\circ} 1,2001$, págs. 1-20.

12 L'Argentina, pensavano in molti, "intimidiva” i vicini, véase Archivo del Ministerio de Relaciones Exteriores del Perú, AMREP, Buenos Aires a Lima, 6 de marzo de 1947; i casi di Uruguay e Cile in Arquivo do Ministério dos Negòcios Estrangeiros do Brasil, AMNEB, Buenos Aires a Rio de Janeiro, 30 de septiembre de 1946. 
1946 - stava diventando esplosiva, proprio mentre la stampa locale si dilungava sugli accumuli di "nobile grano" nei magazzini argentini. Perché non offrirgliene senza chiedere troppo in cambio? Sarebbe stata "una misura d'alta politica e molto conveniente per l'Argentina". Popolo e governo brasiliani l'avrebbero senz'altro ricambiata col loro caldo sostegno negli imminenti, e spinosi, vertici emisferici ${ }^{13}$. Tempo pochi giorni e il ministro degli Esteri brasiliano gli trasmise i suoi profondi ringraziamenti: Buenos Aires avrebbe mandato il grano ${ }^{14}$. Peron aveva fatto i suoi conti. Era pensabile un Blocco latino senza Brasile? Forse Rio non avrebbe aiutato a spezzare il cerchio d'ostilità che ancora circondava l'Argentina? Purché si facesse con prudenza, precisò il ministro brasiliano, attento a non provocare l'ira di Washington, così cara, utile e unita al Brasile ${ }^{15}$.

$\mathrm{Al}$ di là del grano, Peron e suoi mossero per tempo molte tessere su molti piani per comporre il puzzle della Terza Posizione. Dall'Italia alle Americhe, dalla Spagna al Vaticano, nessuna pedina fu trascurata. L'Italia, cui l'Argentina era così legata ed a cui, caldamente incoraggiato dal Papa, Peron prestò aiuti in quantità, fu oggetto di un'audace iniziativa diplomatica, volta per un verso ad avvalorare l'idea che, unica tra le latine d'America, la voce argentina contava nella politica mondiale; e per un altro a conquistare simpatie e riconoscenze quando l'ordine mondiale era ancora fluido e il futuro dell'Italia incerto. Perché escludere che un'eventuale comunità di paesi cattolici e latini la calamitasse? Tant'è: visto lo scoramento italiano per i termini di pace imposti dalle Nazioni Unite, Peron s'appellò a "tutti i governi latini" d'America perché si rivolgessero ai quattro grandi chiedendo loro di stabilire condizioni "giuste ed eque" per l'Italia, in segno di "profondo fervore" verso la "madre spirituale"16.

Ma quel che valeva per l'Italia ancor più s'applicava alla Spagna, la "sentinella" della cristianità dominata da Franco col pugno di ferro. Non foss'altro perché per certi versi la Terza Posizione n'era figlia o figliastra. Alla Spagna nazionalista e cattolica, infatti, doveva l'idea di hispanidad, parimenti ostile ai "fetidi" venti del liberalismo e alle loro "nefaste" filiazioni, socialismo e comunismo; l'idea, cioè, d'una civiltà unica e irriducibile a cavallo dell'Atlantico, cementata da storia, lingua e costumi comuni

13 AMREA, Rio de Janeiro a Buenos Aires, 27 y 28 de febrero de 1946.

14 Cosa e quanto chiedere al Brasile in cambio del grano restò a lungo fonte di tensioni tra $\mathrm{i}$ due paesi e in seno al governo argentino, AMNEB, 10 y 20 de agosto de 1946.

15 AMREA, Rio a Buenos Aires, 6 de marzo de 1946.

16 AMREC, Buenos Aires a Santiago, 24 de mayo de 1946. 
e impregnata di savia cattolica ${ }^{17}$. Tra i due leader e regimi, in più, c'era affinità; anche enormi differenze destinate ad esplodere, è vero, ma un nutrito album di famiglia comune, di cui andavano fieri, essendo militari e cattolici, anticomunisti e antiliberali ${ }^{18}$. Non bastava per gettarsi l'uno nelle braccia dell'altro? Foss'anche, c'era dell'altro. Forse che agli occhi di Peron la Spagna non pativa gli stessi soprusi ch'egli aveva subito durante la guerra? Le stesse pressioni, insulti, umiliazioni per mano dei demoliberali e di Stalin? E perché mai quella Spagna affamata e isolata, di cui tutti attendevano veder sfilare il cadavere, avrebbe rifiutato il salvagente di Peron? Col grano che v'era appeso, naturalmente, e il miraggio d'una comunità di nazioni cattoliche dove la Spagna di Franco avrebbe trovato il suo habitat naturale.

Non sorprende, dunque, ed è risaputo, che tra Peron e Franco nascesse allora un delizioso sodalizio, una tenera intesa presto nota nelle cancellerie come il "piccolo Asse" Buenos Aires-Madrid. Si sa anche che tanto amore non sopravvisse alle focose avances di un nuovo pretendente, alla logica implacabile della guerra fredda, e che il gelo che sopravvenne portò un colpo mortale alle mire europee della Terza Posizione. Ma nel 1946 e per qualche anno ancora Franco ottenne molto da Peron - cibo, crediti, aiuto politico e diplomatico - in cambio, più che altro, dell'adesione spagnola, pur colma di retropensieri, al Blocco latino.

C'è un punto, però, dell'Asse tra Argentina e Spagna, più cruciale degli altri per i destini della Terza Posizione. Proiezione della civiltà cattolica, essa necessitava dell'imprimatur ecclesiastico come dell'aria. E' una faccenda complessa, poiché la Chiesa era ed è varia e sterminata, opaca e poliedrica, e le Chiese di Spagna, Argentina e Roma non parlavano una lingua univoca. Di certo Franco e Peron coltivavano un'idea di Stato cattolico sulle cui virtù dubitavano in molti tra le mura vaticane, ormai consci dei costi d'attaccare la Chiesa al carro d'un particolar regime, per cattolico che fosse. Ma entrambi s'aspettavano una benedizione e l'incondizionata cooperazione della Chiesa in virtù dei loro servigi alla sacra causa. Non solo, ma come ragionevole contropartita per avere riportato Dio nel cuore della vita pubblica contavano d'esercitare indisturbati l'antico diritto regalista di

17 R. de Maetzu e Zacarìas de Vizcarra, forse i due de mayo deri apostoli della hispanidad così intesa, soggiornarono a lungo in Argentina, lasciandovi una ricca eredità intellettuale, véase Zuleta

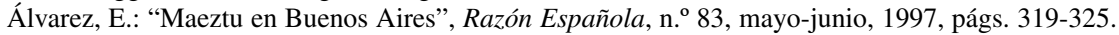

18 Véase Rein, R.: La salvación de una dictadura: alianza Franco-Perón, 1946-1955, CSIC, Madrid, 1995. 
Patronato, che riservando loro la nomina dei vescovi era indigesto al Pontefice $^{19}$.

Non per questo, si sa, la Chiesa si sottrasse ai suoi "doveri" verso Peron e Franco; anzi, contribuì con zelo a cementarne i legami e a nutrire la vaga idea d'una comunità cattolica di nazioni devota alla Santa Sede. Di esempi in tal senso grondano gli archivi: di sacerdoti ambasciatori dell' $h i$ spanidad, di religiosi investiti di delicate missioni politiche. Come José Maria Aguilar, religioso spagnolo e fervente apostolo del franchismo dapprima dinanzi ai riottosi cattolici dell'Uruguay, "infetti" del virus maritainiano, e poi ai ben più benevolenti argentini, decisi a perorare la causa di Spagna nei fori cattolici latinoamericani ${ }^{20}$. O i vescovi argentini, sempre pronti a sguainare la spada dell' hispanidad o a bacchettare i cattolici tiepidi con Franco ${ }^{21}$. Per non dire di Antonio Caggiano, vescovo e cardinale, carissimo a Pio XII e devoto a Peron, invitato, accudito e sfruttato dal governo spagnolo, commosso dal suo accorato tributo alla Spagna franchista in una memorabile intervista ${ }^{22}$.

Ma la Santa Sede? Approvava o riprovava tanta agitazione in nome della cattolicità? E come, perché e per quanto? Il Vaticano era combattuto. Certi fattori, transitori se si vuole, lo spingevano a simpatizzare con i propositi della Terza Posizione, mentre altri, di fondo, gli consigliavano di scoraggiarla. Di certo Pio XII aveva una priorità, ribadita mille volte a chiunque l'incontrasse: contenere e combattere il comunismo, in cui non dubitava risiedesse la maggiore minaccia mai affrontata dalla Chiesa e dal cristianesimo. A tal fine aveva da tempo preso a colmare il profondo fossato scavato dalla storia tra la Chiesa di Roma e gli Stati Uniti, d'accordo con Roosevelt e Truman e col rude ma efficace aiuto del cardinal Spellman. Il mondo era cambiato, come non vederlo? Che senso aveva tornare alle cro-

$19 \mathrm{Su}$ tale, cruciale questione, nei casi di Spagna e Argentina véase Santa Olalla, P.M. de: De la victoria al Concordato. Las relaciones Iglesia-Estado durante el "primer franquismo" (1939-1953), Laertes, Barcelona, 2003 y Zanatta, L.: "La reforma faltante. Perón, la Iglesia y la Santa Sede en la reforma constitucional de 1949", Boletín del Instituto de Historia Argentina y Americana "Dr. Emilio Ravignani”, n. ${ }^{\circ} 20,1999$, págs. 111-130.

20 Archivo del Ministerio de Relaciones Exteriores de Argentina, AMRES, Buenos Aires a Madrid, 21 y 28 de febrero de 1946; sul ruolo del clero nella diplomazia franchista, véase Delgado Gomez-Escalonilla, L.: Diplomacia franquista y política cultural hacia iberoamérica, 1939-1953, CSIC, Madrid, 1988.

21 Véase la spregiudicata difesa della "crociata" spagnola di mons. Barrère, vescovo di Tucumàn, AMRES, Buenos Aires a Madrid, 3 de julio de 1946.

22 L'intervista fu concessa al quotidiano Ya nell'de abril de 1946; véase AMRES, Buenos Aires a Madrid, 17 de enero de 1946; Madrid a Caracas, 2 de mayo de 1946. 
ciate contro liberalismo e democrazia? Ormai la salvezza della cristianità passava da Washington ${ }^{23}$.

Ora, l'intesa con quella grande potenza protestante, patria della libertà di culto e della democrazia liberale, era per la Chiesa una rivoluzione che solo un terribile nemico comune spiegava; ma farla digerire ai cattolici sparsi per il mondo non era così facile ${ }^{24}$. Un po' più in Europa, magari, dove gli americani erano sbarcati da liberatori e lo spettro del comunismo incombeva, ma assai meno in America Latina, dove per gran parte della Chiesa gli Stati Uniti erano gli scaltri imperialisti che foraggiavano le missioni protestanti, minacciavano l'integrità cattolica del continente e meritavano le invettive rivolte loro da tante lettere pastorali ${ }^{25}$. Così non sorprende che in America Latina, più che a Roma, persistessero l'equazione tra cattolicità e latinità e la diffidenza per gli anglosassoni; né che lo spirito della Terza Posizione ne traesse linfa in abbondanza ${ }^{26}$. Tanto più che tra gli effetti della guerra ve n'era un altro, epocale, tutt'altro che irrilevante per i sogni d'un Blocco di nazioni latine e cattoliche. Era sotto gli occhi di tutti, infatti, il peso inedito assunto dalla Chiesa cattolica degli Stati Uniti nel cattolicesimo universale. Forte di 25 milioni di fedeli, traboccava risorse umane ed economiche e superava chiunque in organizzazione; quanto bastava, insomma, perché la sua influenza si sentisse, e forte, sia in Europa che in America Latina ${ }^{27}$. Ma per buona parte del clero e dei governi cattolici in Spagna e nell'America ispanica quell'influenza era una minaccia e un motivo in più per cercare l'unione tra i popoli latini. I missionari cattolici statunitensi in Messico, riferiva un agente spagnolo, fraternizzavano con $\mathrm{i}$ connazionali protestanti; i cattolici messicani erano seccati e chiedevano clero alla Madre Patria ${ }^{28}$. In Sud America c'era poco clero e molta fede, osservava il ministero degli Esteri spagnolo; c'era il rischio che ne profittassero i cattolici di Washington "per influenzare i paesi del Continente, con le conseguenti implicazioni di ordine politico" 29 .

23 Véase Di Nolfo,Vaticano e Stati...; Cooney, J.: The American pope: the life and times of Francis Cardinal Spellman, Times Book, New York, 1984.

24 Véase Kirby, D. (ed.): Religion and the Cold War, Palgrave Macmillan, New York, 2003.

25 Ve ne sono infiniti esempi, dal Messico, véase AMRES, Washington a Madrid, 18 de diciembre de 1944, all'Argentina, véase Zanatta, L.: Perón y el mito de la Nación católica, Sudamericana, Buenos Aires, 1999, págs. 246-250.

26 Non che a Roma tale spirito fosse scomparso; sul rilevante caso del cardinal Ruffini, véase AMREA, Roma a Buenos Aires, 16 de enero de 1946.

27 AMRES, Washington a Madrid, 21 de enero de 1945.

28 AMRES, Washington a Madrid, 18 de diciembre de 1944.

29 AMRES, Dirección de Santa Sede, Informe para la Junta de Política Exterior, abril de 1947. 
Il governo e la Chiesa degli Stati Uniti sapevano che tali e così profondi cambiamenti del panorama politico e religioso generavano tensioni e reazioni; e delle grane che ne derivavano alla Santa Sede, specie nel mondo ispanico. Tanto che per disinnescarle usarono tatto, diplomazia e tonnellate di propaganda anticomunista: un ottimo lenitivo per la diffidenza della Chiesa latinoamericana. Spellman divenne allora il dinamico profeta dell'unità occidentale contro il comunismo, la cerniera tra Stati Uniti, America Latina e Vaticano, al punto che verso il 1947 corse voce della sua nomina a Segretario di Stato. Un'idea, però, che a Washington non piacque: le politiche di Casa Bianca e Vaticano correvano ormai su binari paralleli; perchè provocare i cattolici di Spagna e America Latina con un'ulteriore prova di quell'inedito connubio, per loro così indigesto ? ${ }^{30}$ Perché, anzi, per conciliare l'intesa col Papa e il buon vicinato con l'America Latina, non aprire un'Ambasciata in Vaticano? Ci pensarono in molti, al Dipartimento di Stato, seppur senza successo: i rapporti con l'America Latina se ne sarebbero giovati ${ }^{31}$, anche a danno del fascino d'un Blocco latino.

All'indomani della guerra, comunque, fioccavano segni tali da indurre il Papa a osservare con discreta benevolenza l'idea d'un Blocco cattolico e Peron a giudicarlo un tacito compagno di viaggio. Il fatto è che ancora nel 1946, benché montassero le tensioni tra Washington e Mosca e maturassero i semi della guerra fredda, in Vaticano non s'era certi che gli Stati Uniti avrebbero preso con fermezza la guida dell'Occidente e acquartierato truppe in Europa. Nel dubbio, sconvolta dalla repressione anticattolica d'oltrecortina, la Santa Sede non disprezzava l'eventualità che le nazioni cattoliche le facessero quadrato intorno. La situazione non era cambiata granché da quando, nel 1944, dialogando con l'incaricato d'Affari argentino, Pio XII aveva evaso l'interrogativo sulla capacità degli angloamericani di contrastare i sovietici ${ }^{32}$. Né da quando, nel 1945, aveva lodato Salazar, rifiutatosi di scambiare la neutralità con l'ammissione alle Nazioni Unite, e condiviso il timore per le pretese alleate d'esportare il demo-liberalismo laddove regnavano i più pii tra i regimi ${ }^{33}$.

La simpatia vaticana per la tendenza a fare gruppo delle nazioni cattoliche e quella delle Chiese d'Argentina e Spagna per le ambizioni di $542-544$.

30 Parsons a Bowling, 26 de septiembre de 1947, en Di Nolfo, Vaticano e Stati..., págs.

31 Tittman a Stettinius, 26 de junio de 1945, en Di Nolfo, Vaticano e Stati..., págs. 458-459.

32 AMREA, Roma a Buenos Aires, 3 de diciembre de 1944.

33 Così riferì l'Ambasciatore portoghese, AMNEP, Roma a Lisbona, 10 de abril de 1945. 
Peron e l'ideale dell'ispanità, rischiavano però d'irritare gli Stati Uniti, decisi a tutto pur di raggruppare l'intera America Latina sotto la loro ala in nome del panamericanismo. Mai e poi mai, insomma, avrebbero tollerato Blocchi autonomi in America Latina e men che meno qualora avessero ambito ad estendersi all'Europa, in spregio della dottrina Monroe ${ }^{34}$. E' vero, Peron era stato eletto e alla Casa Bianca sapevano di dover blandirlo per ricondurlo all'ovile senza turbare l'unità americana. Ma non lo amavano e lo tenevano nel mirino, convinti, come tanti nelle Americhe ${ }^{35}$, ch' egli non si facesse scrupoli pur di spingere i vicini a spalancare le porte alla Terza Posizione. Peron, ne erano certi, voleva "formare un blocco" di nazioni latinoamericane ${ }^{36}$.

\section{La Terza Posizione alla prova della Guerra Fredda}

Il 4 giugno 1946 Peron entrò alla Casa Rosada. La data la diceva lunga: decorrevano tre anni esatti dal golpe ch'egli celebrava come l'inizio della sua rivoluzione; l'inizio dell'era fascista, per i suoi avversari. La Terza Posizione n'era parte organica, anima e sostanza; altro che slogan ${ }^{37}$. Peron, dunque, l'avrebbe perseguita a ogni costo. In essa si rispecchiava il nucleo ideale del justicialismo, parimenti antiliberale e anticomunista, e l'irremovibile convinzione di Peron che solo un'Argentina emancipata dai potentati stranieri e forte d'una possente industria avrebbe avuto la libertà di stabilire se, come e quando partecipare alla nuova guerra che s'annunciava. Novello San Martìn, egli avrebbe allora portato il suo Vangelo al di là delle frontiere per "liberare" e "unire" l'America Latina ${ }^{38}$. Era un'idea ambiziosa. Anche perché al netto dell'euforia postbellica, l'economia argentina non si prestava a tanti sogni. E poi c'erano cuori da conquistare e timori da fugare. La Terza Posizione doveva suonare benevola, idealista, pacifista, perché non paresse uno strumento di conquista, un cuneo per dividere. $\mathrm{Ci}$ voleva cautela per sedurre i fratelli latini senza stuzzicarne

34 Schwartzberg, S.: Democracy and U.S. policy in Latin America during the Truman years, University press of Florida, Gainesville, 2003.

35 In Brasile più che mai, véase AMNEB, Buenos Aires a Rio de Janeiro, 30 de septiembre de 1946.

36 AMREC, La Paz a Santiago, 28 de mayo de 1946.

37 Così, tra i tanti, la definisce Page, J.: Perón. Una biografía, Sudamericana, Buenos Aires, 2005, pág. 224.

38 Così amava dire Peron, véase AMREP, Buenos Aires a Lima, 30 de junio de 1947. 
sospetti e gelosie, ma anche fermezza per coltivare gli interessi economici nazionali e slancio per tener viva la fiamma rivoluzionaria e antimperialista tra le masse. Tutto e il suo contrario, insomma: diplomazia, economia, ideologia; sorrisi e minacce, bastoni e carote. Da qui il doppio, triplo, quadruplo discorso di Peron, leader istrionico e grande affabulatore, fonte d'amori e timori, equivoci e speranze, e della fitta trama di contraddizioni in cui s'impantanò la Terza Posizione.

Per cominciare, va da sé, c'era Washington da calmare; e proprio su ciò cui Peron più puntava, il famoso Blocco latino. C'erano elementari considerazioni geopolitiche e mille altri buoni motivi perché, placatosi il ciclone Braden, tra i due paesi tornasse un po' di sereno ${ }^{39}$. Valeva per Peron, ma anche per Truman ${ }^{40}$, il cui edificio panamericano non sarebbe mai stato solido senza il pilastro argentino e finché Peron l'avesse corroso con l'idea del "suo" Blocco. Tanto che a Buenos Aires mandò, colmo di buone intenzioni, l' ambasciatore Messersmith, con cui Peron tutto fece e tutto disse per fugare i sospetti sui suoi piani; fin troppo, poiché tanto maldestro fu nel farne l'anti-Braden che molti, a Washington, sospettarono servisse Peron più di Truman ${ }^{41}$. A Messersmith, Peron si presentò rassicurante, senza grilli per la testa né fantasie di Blocchi latini ${ }^{42}$. Certo, gli disse, sperava che il mondo non si spaccasse in blocchi; ma qualora, come pareva, fosse accaduto, l'Argentina, cui ripugnava il comunismo e su cui era certo gravassero le brame sovietiche, sarebbe stata con l'Occidente e l'emisfero americano. L'inveterato isolazionismo argentino, aggiunse, era stato un errore. Nulla, insomma, doveva insospettire Messersmith; perfino al costo di liquidare la feroce rivolta di La Paz contro il presidente Villarroel, il suo alter ego boliviano d'un tempo, come una normale insurrezione popolare contro un leader $\operatorname{arbitrario}^{43}$.

Ma non tutto era rose e fiori. Peron, infatti, aveva in mano una carta; un asso da giocare con prudenza ma assai utile per incendiare o quietare $\mathrm{i}$ rapporti con Washington: molti argentini, disse, specie dei suoi, erano

39 Véase Escudé, Gran Bretaña, Estados Unidos...

40 Com'era evidente a tanti, véase AMREP, Buenos Aires a Lima, 6 de junio de 1947.

41 Sullo scarso tatto di Peron e della sua stampa convenne lo stesso Messersmith, véase AMNEB, Buenos Aires a Rio de Janeiro, 8 de agosto de 1946; AMREP, Buenos Aires a Lima, 21 de diciembre de 1946; tanti elogi argentini a Messersmith gli erano dannosi, commentò l'Ambasciatore statunitense a Rio, véase AMREA, Rio de Janeiro a Buenos Aires, 11 de enero de 1947. $171-181$.

42 Van Der Karr, J.: Perón y los Estados Unidos, Vinciguerra, Buenos Aires, 1990, págs.

43 Ibidem, pág. 177; véase anche Zanatta, Bolivia, Peron... 
antiamericani. Non ch'egli, pur comprendendoli, ne condividesse il sentimento, ma occorreva tenerne conto. Messersmith, però, non ne dubitasse: appena il tempo di sanare il contenzioso tra i due paesi ed egli, coi suoi taumaturgici poteri, avrebbe fatto sparire ogni traccia di quell'astio. Ma davvero egli era pronto a riporre il sogno d'una comunità latina di nazioni se solo gli Stati Uniti avessero fatto ammenda e liquidato le ritorsioni imposte all'Argentina? Non proprio; era un bluff, insomma, al massimo un pio desiderio: come placare il demone antiamericano dopo averlo così tanto evocato? Altrove ma allo stesso tempo, difatti, il suo linguaggio non mutò; né il governo argentino accantonò i suoi progetti, né quello di Washington ne prese per oro colato le professioni di fede occidentale. I due eccezionalismi, insomma, non smisero di competere e la Terza Posizione non andò in soffitta. Parlando ai futuri addetti operai, per dire, Peron li fregiò del titolo di "soldati di conquista"; voce dei suoi fedeli, quei sindacalisti pronti a sparpagliarsi per il mondo col passaporto diplomatico in tasca dovevano espandere la rivoluzione, diffonderne la verità ${ }^{44}$.

Non c'era angolo d'America, d'altronde, da cui non salissero gli echi dei contrasti tra Washington e Buenos Aires. Dal Perù, dove il governo a corto di grano si dimenava per ridurre gli esosi prezzi pretesi da Buenos Aires cercando farina a Washington e pur di non perdere un credito dell'Exim Bank resisteva alle proposte argentine d'investire nel carbone ${ }^{45}$. Dalla Bolivia, crocevia d'una lotta senza esclusioni di colpi, dove la Casa Bianca vedeva all'opera il disegno peronista d'una "lega Austral" ed altri una sfrenata gara per lo stagno tra gli Stati Uniti e il loro maggior rivale economico in America Latina ${ }^{46}$. Dal Brasile, dove l'ombra di Washington incombeva sull'annoso contenzioso dello scambio tra grano argentino e gomma brasiliana ${ }^{47}$. Dappertutto, insomma.

Non stupisce, dunque, che tra Argentina e Stati Uniti corressero due binari, sgombro l'uno, irto d'insuperabili ostacoli l'altro, quello su cui

44 AMNEP, Buenos Aires a Lima, 3 de septiembre de 1946.

45 AMNEP, Buenos Aires a Lima, 16 de julio de 1946 y 25 de marzo de 1947; Dorn, G.J.: “'Exclusive Domination' or 'Short Term Imperialism': The Peruvian Response to U.S. - Argentine Rivalry, 1946-1950”, The Americas, v.61, n. ${ }^{\circ}$, julio, 2004, págs. 81-102.

46 Così la vedevano i circoli diplomatici, véase AMRES, Washington a Madrid, 30 y 31 de julio de 1946. Intimi di Peron, gli spagnoli toccarono con mano la pesante ingerenza argentina in Bolivia, véase AMRES, José Gallostra a Ministro, 24 de diciembre de 1946; AMREP, Buenos Aires a Lima, 6 de marzo de 1947.

47 Per motivi ora politici ora economici Buenos Aires lesinava il grano al Brasile; gli Stati Uniti colmarono il gap, véase AMREA, Rio de Janeiro a Buenos Aires, 6, 13 y 15 de noviembre de 1946; AMNEB, Buenos Aires a Rio de Janeiro, 13 de septiembre de 1946. 
viaggiava la Terza Posizione. Con Washington il tempo volge al bello, confidava il ministro argentino Bramuglia nel febbraio 1947; e Messersmith, di rimando: presto tutto sarà risolto tra noi e insieme potremo formare il blocco americano caro a Peron ${ }^{48}$. Gratta gratta, però, c'era del marcio. L'Ambasciatore desiderava certo che il suo governo confidasse in Peron, ma perplessità e dubbi ne aveva anche lui. Per ora, diceva nell'agosto del 1946, tante parole e pochi fatti; e in novembre: Peron è in buona fede, ma chi lo consiglia no ${ }^{49}$. Quel che molti temevano a Washington lo disse senza girarci intorno al suo collega argentino l'Ambasciatore statunitense in Brasile, William Pawley: io, il mio governo e la classe dirigente del mio paese pensiamo che Peron voglia formare un blocco latinoamericano ostile agli Stati Uniti ${ }^{50}$.

Esagerazioni? Calunnie? Banali scuse per intimidire un irruente rivale? Può darsi, ma non c'è dubbio che al di là del miele profuso, Peron tirasse dritto per la sua strada. Proprio allora, difatti, nei primi mesi del 1947, quando ormai si stagliavano i contorni della guerra fredda e s'avvicinava il concepimento del nuovo sistema panamericano nella Conferenza di Rio, Peron si preparava ad illustrare al mondo la Terza Posizione. Le lunghe, chiassose, pompose e costose missioni europee e americane del senatore Molinari dovevano dissodarle il terreno. Quintessenza della "nuova Argentina", spavaldo e sopra le righe, latore di promesse e minacce, lusinghe e dileggi, Molinari suscitava ironie e timori; ma anche brame e speranze, con la sua valigia colma del miraggio di buoni affari: trattati commerciali, nuove industrie e banche, vie commerciali servite dalla fiammante flotta mercantile argentina e quant'altro ${ }^{51}$.

E d'altronde, qualora le trame militari, le pressioni economiche e i corteggiamenti sindacali non fossero bastati a confermare che gli abboccamenti con gli Stati Uniti non avevano distolto Peron dall'idea d'un Terzo blocco, c'era il suo linguaggio a fugare dubbi: un linguaggio assai diverso allorché invece che in compagnia dei sospettosi emissari di Washington si trovava "in famiglia", a quattr'occhi con i suoi veri o presunti soci. Come

48 L'Ambasciatore spagnolo Bulnes incontrò entrambi, véase AMRES, Buenos Aires a Madrid, 7 de febrero de 1947.

49 AMREC, Buenos Aires a Santiago, 13 de agosto y 14 de noviembre de 1946.

50 AMREA, Rio de Janeiro a Buenos Aires, 11 de enero de 1947, Pawley e Messersmith si tenevano in stretto contatto.

51 AMREC, Lima a Santiago, 14 de marzo de 1947; Buenos Aires a Santiago, 25 de abril de 1947; AMRES, Buenos Aires a Madrid, 19 de junio de 1947; AMREP, Buenos Aires a Lima, 4 de junio de 1947. 
Demetrio Carceller, falangista di grido e gerarca di Spagna, dinanzi al quale, solleticandone il furibondo anticomunismo, Peron se la prese con i tentennamenti degli "anglosassoni" verso Mosca: l'ennesima ragione per sottrarsi all'orbita dei due imperialismi emersi trionfanti dalla guerra ${ }^{52}$.

Certo, il suo era un governo variegato, con i suoi oltranzisti e le sue colombe. La Terza Posizione, per dire, assumeva un' aria torva e minacciosa in bocca al ministro Miranda, deciso a resuscitare una sorta di vicereame economico ${ }^{53}$, o di qualche militare esaltato che già sognava di rivoluzionare la mappa del continente ${ }^{54}$; e un aspetto più innocuo in quella di Bramuglia, che per esperienza, tatto e temperamento badava a non attizzare ulteriori sospetti e ad evitare che la Terza Posizione si trasformasse in un luogo solitario e inospitale, in un imbuto nero tra i due blocchi. Ma tali cautele presto crollarono, e Bramuglia con loro, sotto il peso dell'aggressivo antiamericanismo dell'ala sindacale, dell'onnipotente ostilità di Eva Peron e dell'intrinseca tensione tra Terza via e via panamericana ${ }^{55}$. Una tensione evidente ogniqualvolta qualcuno si sforzava di spiegare che la Terza Posizione serviva ad affermare la "personalità" latina dinanzi agli anglosassoni, e non a contrastare gli Stati Uniti ${ }^{56}$. Ragionevole o meno, infatti, a Washington quella spiegazione non andava giù: distinguere tra civiltà diverse nello stesso continente non inibiva l'unità contro il nemico comune? E poi, quel distinguo tra Nord e Sud, anglosassoni e latini, protestanti e cattolici, non portava linfa al nazionalismo latinoamericano, la cui bestia nera era "l'imperialismo yankee"? Spiegata col ringhio o col sorriso, la Terza Posizione non cambiava: il governo argentino, chiarì Bramuglia all'Ambasciatore spagnolo, desiderava che le nazioni latine d'America e d'Europa stabilissero un legame tale da scongiurare la divisione del mondo in due soli blocchi, anglosassone l'uno, slavo l'altro ${ }^{57}$. Non aveva torto, dunque, Peron, ma neanche di che meravigliarsi, quando lungi dal celebrare le sospirate dimissioni di Braden, nel giugno 1947, lamentò la strenua

52 AMRES, Buenos Aires a Madrid, 19 marzo 1947.

53 Review of the River Plate, 10 de enero de 1947; AMNEB, Buenos Aires a Rio, 9 de diciembre de 1946.

54 Il clamoroso caso del colonnello Carlés, addetto militare a La Paz, in AMREC, La Paz a Santiago, 19 de marzo y 27 de agosto de 1947.

55 Su come Eva Peron si liberò di Bramuglia, véase AMREC, Buenos Aires a Santiago, 5 de septiembre de 1949; sulle origini del loro conflitto Page, Peron..., pág. 155.

56 Così la presentò Bramuglia agli Ambasciatori di Spagna, Francia e Italia, AMRES, Buenos Aires a Madrid, 17 de mayo de 1947.

57 Ibídem. 
ostilità statunitense ai suoi sforzi di affermare la "personalità" argentina in America del Sud ${ }^{58}$.

Non per questo, va da sé, Peron ammainò la bandiera. S'è visto: aveva frecce al suo arco e la Terza Posizione era il cuore, non il ripostiglio del suo pensiero. E poi l'idea d'una comunità latina non era nuova né balzana per governi e popoli dell'America ispanica e il suo vangelo sociale suonava a festa nelle orecchie di tanti operai latinoamericani, ben più sfortunati dei compagni argentini. Né gli Stati Uniti erano così amati e riveriti a sud del Rio Grande, dove in tanti si sentivano abbandonati nonostante la lealtà mostrata in tempo di guerra. Per Peron, insomma, c'era terra fertile in America e spazio per giocare il gioco caro ai piccoli in un mondo bipolare: perché non alzare il prezzo della propria amicizia, magari agitando il terribile spettro comunista?

Il vertice di Rio, nell'agosto 1947, dove Truman contava di stringere le maglie della difesa emisferica contro il comunismo, era un'occasione d'oro. "Avranno un bel da fare se credono che ci accorderemo", minacciava Peron, furioso per la rimozione di Messersmith e deciso a sfidare la politica "assorbente" di Washington". Tanto più che il piano statunitense non piaceva a tutti: vari governi, come l'argentino, temevano per la sovranità ${ }^{60}$. Così, per cercare spalle e fissare una strategia comune in vista di Rio, Molinari e Bramuglia riunirono gli Ambasciatori latinoamericani; pur di convincerli, Molinari era pronto a elargire crediti nella sua ennesima, imminente missione continentale ${ }^{61}$. Ma se Peron confidava nei venti favorevoli, c'era chi l'avvertiva di fare attenzione a quelli contrari, non meno forti e pericolosi. Ci accusano di imperialismo, osservò allarmato lo Stato Maggiore dell'Esercito quando nel Congresso brasiliano risuonarono le violente invettive contro l'Argentina del deputato Flores da Cunha; e di lucrare sul grano violando gli accordi internazionali. Non arriveremo a Rio menomati, domandava il generale Sosa Molina? L'Argentina non sarebbe stata oggetto d'astio e sospetto proprio da parte di coloro che ambiva a trascinare nella sua avventura? E' tutto ok, rispose Bramuglia, ma il nodo c'era, e bello grande ${ }^{62}$.

58 AMRES, Buenos Aires a Madrid, 5 de junio de1947.

59 AMRES, Buenos Aires a Madrid, 5 y 6 de junio de 1947.

60 Come notò l'Ambasciatore di Spagna, che chiese cautela alla stampa spagnola, véase AMRES, Buenos Aires a Madrid, 29 de mayo de 1947.

61 AMREP, Buenos Aires a Lima, 6 de junio de 1947.

62 AMREA, Sosa Molina a Bramuglia, 12 de junio de 1947; Bramuglia a Sosa Molina, 16 de junio de 1947 . 
Poiché a Rio come altrove Peron voleva dare a Truman del filo da torcere e far suonare la grancassa della Terza Posizione, niente pareva meglio che introdurvi uno dei suoi cavalli di battaglia ${ }^{63}$; che smuovervi mari e monti perché in barba all'Onu i fratelli ispanici schiudessero le porte della comunità latina al generale Franco e riaprissero quelle delle loro Ambasciate a Madrid ${ }^{64}$. Come aveva fatto Peron, sanando quel che gli pareva un indecente castigo al più cattolico e anticomunista dei governi comminato dal tossico connubio tra potenze comuniste e democratiche ${ }^{65}$. Si sarebbe così ravvivata la fiamma ispanista che albergava repressa in tanti animi e puntellata la sponda europea della Terza Posizione, ancorando ancor più saldamente la Spagna all'Argentina. Era una corsa contro il tempo, Peron lo sapeva, prima che a Franco si aprisse la via di Washington, dove la "lobby spagnola" stava conquistando deputati e militari ma si arenava ancora sugli scogli di Truman e degli alleati europei ${ }^{66}$. Da tempo, peraltro, Peron lavorava per sdoganare Franco. Non solo mandandogli un Ambasciatore, accolto a Madrid come un Messia ${ }^{67}$, ma anche imponendone la causa alle cancellerie americane, magari per fare ammettere la Spagna al Congresso Postale di Parigi ${ }^{68}$; come Pio XII, d'altronde, autore d'un passo analogo presso Irlanda e Svizzera perché protestassero contro l'esclusione spagnola ${ }^{69}$.

A Rio la questione s' annunciava complessa. Pareva certo, infatti, che la Casa Bianca mirasse a saldarvi un'alleanza anticomunista sudamericana; a farne l'occasione, insomma, per trapiantare nelle Americhe la dottrina del containment. Dato l'anticomunismo dei governi di Rio e Buenos Aires, gli Stati Uniti speravano che quella fosse la chiave per indurli a cooperare e per cementare l'unità emisferica ${ }^{70}$. Il vertice tra Peron e il presidente brasi-

63 Bramuglia, da vecchio socialista, l'aveva meno a cuore, véase AMRES, Buenos Aires a Madrid, 21 de mayo de 1947.

64 Molinari ricevette precise istruzioni in tal senso; AMRES, Buenos Aires a Madrid, 19 de junio de 1947.

65 Come Peron amava dire, véase AMRES, Buenos Aires a Madrid, 30 de septiembre de 1947.

66 Viñas, A.: En las garras del Águila. Los pactos con Estados Unidos, de Francisco Franco a Felipe González (1945-1995), Crítica, Barcelona, 2003, págs. 57-65.

67 AMRES, Buenos Aires a Madrid, 18 de enero de 1947.

68 Véase Madrid a Buenos Aires, 22 marzo 1947, Buenos Aires a Madrid, 3 de abril de e 19 de mayo de 1947.

69 Nemmeno stavolta la Spagna fu ammessa, ma il voto l'indusse all'ottimismo sui nuovi venti che spiravano in America Latina, véase AMRES, Madrid a Buenos Aires, 10 de marzo de 1947 e Buenos Aires a Madrid, 2 de junio de 1947.

70 AMREC, Buenos Aires a Santiago, 11 de marzo de 1947; AMRES, Madrid a Buenos Aires, 22 de marzo de 1947; Buenos Aires a Madrid, 26 de marzo de 1947. 
liano Dutra, disposto a mediare tra Argentina e Stati Uniti, parve spianare la via a tale scenario ${ }^{71}$.

Alla fine però le cose vi andarono altrimenti e a farla da padrone fu la difesa emisferica. Era presto e non c'era ancora il clima giusto perché l'anticomunismo unisse la regione. Perché in America Latina la minaccia comunista non incombeva come in Europa ${ }^{72}$; perché c'era chi vi scorgeva il cavallo di Troia dell'interventismo statunitense e chiedeva garanzie o contropartite; perché alcuni governi non ne volevano sentir parlare; ma anche perché neppure sull'anticomunismo, dove si sarebbe detto che fossero sulla stessa lunghezza d'onda, c'era intesa tra Argentina e Stati Uniti. Anzi, proprio su quel terreno avvampava la competizione tra la Terza Posizione e Washington e presto la strada di Peron si sarebbe divaricata perfino da quella di Franco e Pio XII.

Per Peron l'eventualità che a Rio s'approvasse una risoluzione anticomunista aveva un particolare valore politico. Si sarebbe in tal modo aperta la via alla normalizzazione tra i paesi americani e la Spagna e il suo regime avrebbe troneggiato al crocevia tra vecchio e nuovo mondo latino. Che così fosse era chiaro un po' a tutti ${ }^{73}$. Franco ne fu così incoraggiato da adoperarsi perché il vertice affrontasse la questione spagnola ${ }^{74}$. Ma per Peron l'anticomunismo non si prestava a far da brodo di coltura del panamericanismo. Semmai era un terreno tipico della Terza Posizione; un ambito, cioè, in cui le nazioni latine si distinguevano dagli Stati Uniti e dov'egli s'ergeva a leader e modello. Non aveva dubbi, infatti, e non fece che ripeterlo alle osannanti folle peroniste, che il suo justicialismo fosse l'unica terapia efficace per estirpare il cancro comunista dalle menti della classe operaia; quel cancro che il capitalismo anglosassone produceva a suon di sfruttamento e bieco materialismo ${ }^{75}$.

Come pensare, dunque, a un fronte anticomunista sotto l'ala di coloro cui addebitava la responsabilità dell'infezione comunista in America

71 AMRES, Madrid a Buenos Aires, 16 de mayo de 1947; Buenos Aires a Madrid, 23 de mayo de 1947.

72 Bethell, L., Roxborough, I. (ed.): Latin America between the Second World War and the Cold War, 1944-1948, Cambridge University Press, Cambridge, 1992.

73 AMRES, Buenos Aires a Madrid, 10 de mayo de y 24 de junio de 1947; Madrid a Buenos Aires, 21 de mayo de 1947.

74 AMRES, Madrid a Buenos Aires, 29 de mayo de 1947; Buenos Aires a Madrid, 2 de junio de 1947.

75 Peron si espresse un'infinità di volte in tal senso;AMREC, Buenos Aires a Santiago, 11 de marzo de 1947; véase "El Primer Magistrado habló ante un grupo de periodistas extranjeros", 1 de abril de 1948 . 
Latina? Il comunismo, amava dire Peron, si combatteva col Vangelo in mano, realizzando le encicliche sociali dei pontefici ${ }^{76}$, dando soldi, casa, cibo, diritti e dignità agli operai, colpendo i privilegi delle classi dirigenti e l'arbitrio del grande capitale straniero; e naturalmente organizzando i lavoratori in un grande sindacato impregnato di "dottrina nazionale". Come non vedere, tuonava Peron, il successo della sua politica? Che la classe operaia argentina era quella che meglio stava in America Latina e più rifuggiva le bandiere rosse? Tanto, soleva aggiungere, ch'egli non aveva alcun bisogno di leggi anticomuniste, o di spedire a casa l'Ambasciatore sovietico, come altri facevano in ossequio ai caldi suggerimenti di Washington. D'accordo, allora, sul fronte anticomunista, ma sotto la sua guida e nel segno di justicialismo e Terza Posizione. Perciò ne aveva proposto uno a Cile e Brasile, i vicini insieme più ostici e soggetti all'influenza comunista; e perciò entrambi avevano declinato l'invito, cui aderivano ora che a farlo era Truman: un brutto colpo per Peron e un altolà alle sue ambizioni ${ }^{77}$. Non solo perché confermava che Washington aveva buoni argomenti, ma anche che i suoi vicini erano pronti a sfruttarli per tenerlo a bada, giudicandone l'ideologia inguaribilmente espansionista.

La ricetta anticomunista, pensava Peron, era un'esclusiva della Terza Posizione. Ed era tutt'uno col suo nazionalismo radicale, la sua base operaia, il suo ethos rivoluzionario che ne facevano un boccone indigeribile per il panamericanismo statunitense; un virus letale. Tanto più che a Washington aleggiava il timore che la longa manus sovietica, troppo distante dalle Americhe per lasciarvi impronte, si acquattasse tra le pieghe dei tanti e fiorenti movimenti nazionalisti di cui il peronismo era l'archetipo ${ }^{78}$. La stessa, battente invocazione peronista della giustizia sociale che spandeva l'eco della Terza Posizione tra le masse d'America e talvolta d'Europa, era fonte di sospetti e resistenze. Quanti governi, perfino amici di Peron, ne respinsero con sdegno gli addetti operai, accusandoli di sobillare lo spirito rivoluzionario dei mansueti lavoratori locali ${ }^{79} \mathrm{E}$ il turbamento franchista per le infuocate parole di Eva Peron agli operai di Barcellona? ${ }^{80}$ Non tradivano,

76 AMREC, Buenos Aires a Santiago, 18 de noviembre de 1948.

77 AMREP, Buenos Aires a Lima, 9 de octubre de y 15 de noviembre de 1947.

78 Welles, S.: "Necesidad de una doctrina interamericana sobre reconocimientos", La Razón, La Paz, 24 de mayo de 1944.

79 Moltissimi; il caso emblematico del Portogallo in AMNEP, Repartiçao dos Negocios Politicos, 11 de julio de 1952; tanti li giudicavano controproducenti, véase AMRES, Buenos Aires a Madrid, 16 de enero de 1948; AMREC, Buenos Aires a Santiago, 27 de agosto de 1948.

80 AMREC, Buenos Aires a Santiago, de julio de 1947. 
forse, uno strisciante egemonismo argentino? Si capisce, dunque, tanta circospezione intorno alla Terza Posizione; e si comprende, altresì, che Peron, esasperato, si rivolgesse ai popoli saltando i governi, cui parve dunque ancor più minaccioso, coi suoi intrighi e le sue ingerenze ${ }^{81}$. Che l'anticomunismo fosse uno scoglio tra Peron e la comunità occidentale e ch'egli mirasse a farne un punteruolo per scavare la via della Terza Posizione tra le muraglie della guerra fredda, lo confermavano i suoi ambivalenti rapporti con Mosca. Tipici, in fondo, d'un attore ambizioso costretto in una rigida cornice bipolare, ma a doppio taglio. Utili, forse, da agitare verso gli Stati Uniti per contrastarne l'ostilità, ma tali, di certo, da contrariare i soci più cari e vitali, specie Spagna e Santa Sede, ai quali Peron si proponeva come alfiere d'un anticomunismo più duro e cristallino di quello statunitense ${ }^{82}$. Era, il suo, un gioco esplicito e rischioso; comprensibile, però, alla luce della sua certezza che tra i Blocchi vi fosse spazio e che il tempo lo favorisse: l'imminente, inevitabile guerra, pensava, gli avrebbe dato ragione e condotto alla sua corte quanti desideravano decidere da sé se e quando entrarvi. L'Unione Sovietica, in sintesi, era nemica dell'Argentina, garantì Peron a Messersmith; a meno che, precisò il ministro Lagomarsino, gli Stati Uniti non l'obbligassero altrimenti; erano stati loro, ricordò Bramuglia, che isolandola l'avevano indotta a stabilire rapporti con Mosca ${ }^{83}$. Ora con Washington pareva tornato il bel tempo, perciò Peron inveiva contro comunisti e sovietici; senza rompere, però, perchè il tempo e gli umori, si sa, cambiano ${ }^{84}$.

Ci voleva misura, però, per non tirare troppo la corda con gli Stati Uniti e i partner latini; e anche per non prestarsi ai disegni di Mosca. Correva voce, infatti, vera o falsa poco importa, che l'idea di un Blocco latino ficcato come un bastone tra le ruote statunitensi piacesse ai sovieti$\mathrm{ci}^{85}$; certo, un po' meno ai comunisti argentini e cileni, italiani e francesi, ai cui occhi il peronismo restava il diavolo, ma assai più ai brasiliani, il cui leader indiscusso, Luis Carlos Prestes, coprì presto Peron di elogi e prof-

81 Peron ne parlò con l'Ambasciatore spagnolo, véase AMRES, Buenos Aires a Madrid, 17 de julio de 1947; sulle ingerenze, per il caso cileno véase Machinandiarena de Devoto, L.: Las relaciones con Chile durante el peronismo, 1946-1955, Lumière, Buenos Aires, 2005.

82 AMRES, Buenos Aires a Madrid, 30 de septiembre de 1947.

83 Van Der Karr, Perón y los Estados Unidos..., págs. 162 y 175; AMRES, Buenos Aires a Madrid, 8 de marzo de 1947.

84 AMREC, Buenos Aires a Santiago, 11 de marzo de 1947; AMREP, Buenos Aires a Lima, 15 de noviembre de 1947.

85 AMRES, Madrid a Buenos Aires, 14 de agosto de 1947. 
ferte ${ }^{86}$. Un'affinità elettiva tra uomini d'arme quali erano Prestes e Peron? Un occulto piano di Mosca? Chissà. Sta di fatto che la guerra fredda rendeva porosi i confini della Terza Posizione e tendeva a farne un autobus colmo di viaggiatori d'ogni tipo, che salivano se serviva salvo scenderne al primo intoppo.

\section{Remare contro corrente. Il mondo diviso e la Terza Posizione}

Nel luglio 1947, mentre una pesante saracinesca scendeva in mezzo all'Europa e si stagliavano i contorni dei Blocchi, Peron annunciò urbi et orbi la Terza Posizione. Quel che più temeva e intendeva scongiurare stava avvenendo: un mondo bipolare, pensava, avrebbe fatto calare una pietra tombale sulla sovranità latinoamericana e costretto l'Argentina ad accomodarsi ai margini dell'impero di Washington. La guerra gli pareva questione di tempo: i militari statunitensi, diceva, scalpitavano per colpire l'Unione Sovietica finché era debole, e solo Truman li tratteneva, per ora ${ }^{87}$. Era perciò giunta l'ora di chiarire che pur parteggiando per l'Occidente, l'Argentina justicialista non intendeva assoggettarsi ad alleanze che ne limitassero sovranità e libertà d'azione in caso di guerra; di indicare a milioni di figlie e figli della civiltà latina e cattolica che in quel mondo costretto a schierarsi di qua o di là dal precipizio esisteva un'altra via, una Terza Posizione, quella dell' Argentina di Peron ${ }^{88}$.

In parte, dunque, Peron reagì alla piega presa dall'ordine mondiale; ma solo in parte, poiché nulla di quanto fece rispose al caso o alla fretta. In fondo, infatti, quel che disse si sapeva lo pensasse e giunse al culmine di un'intensa preparazione, in cui si avvalse di tante armi: dal grano alla luna di miele con la Chiesa, dalle cerimoniose missioni di Molinari al corteggiamento delle Ambasciate dei paesi latini; per non dire, nel giugno 1947, del tour europeo di Eva Peron, la più chiassosa rampa di lancio della Terza Posizione ${ }^{89}$. Un viaggio riuscito a metà. Poco incoraggiante, insomma, per la "gamba" europea della Terza Posizione; Spagna a parte, va da sé, poiché da lì, rifiutato l'invito statunitense a soprassedere, cominciò il periplo di Eva, suggello del "piccolo Asse" Madrid - Buenos Aires e "solidale espres-

86 AMREA, Rio de Janeiro a Buenos Aires, 4 de febrero de 1947.

87 AMRES, Madrid a Buenos Aires, 17 de julio de 1947.

88 AMRES, Madrid a Buenos Aires, 8 de junio de 1947.

89 Così l'intese l'Ambasciatore del Perù, AMREP, Buenos Aires a Lima, 4 de agosto de 1947. 
sione" justicialista "di cristianità, pace e giustizia sociale" ce, fu un flop. A cominciare dall'Italia, dove Eva trovò un governo tiepido e i descamidados locali a urlarle improperi sotto le insegne del partito comunista $^{91}$, proseguendo con la tappa "estorta" in Francia ${ }^{92}$, paese in preda, disse, a "comunistizzazione avanzata", dove affermò o millantò d'aver dovuto "comprare" la stampa pur di addolcirne i toni, e finendo in Portogallo, dove le parve regnare "la più implacabile delle dittature". Il tutto condito dalla ruvida udienza con Pio XII, urtato dalla fede zelante, esibita e sospetta di Eva, tutta dedita a strappargli decorazioni per sé e il marito; un Papa già circospetto per le evidenti tracce di regalismo nel governo di Peron ${ }^{93}$.

Il 6 luglio, infine, Peron parlò al mondo e la sua macchina propagandistica ne amplificò l'eco all'infinito, specie nelle Americhe e in Vaticano, dove il discorso giunse accompagnato da una nota diplomatica ${ }^{94}$. La Terza Posizione era in orbita, finalmente, benché ammantata di toni candidi e pacifisti che ai più caustici, come l'Ambasciatore di Spagna, parvero "una notevole evoluzione" rispetto a quelli assai più schietti impiegati poc'anzi da Peron davanti ai suoi parlamentari. Quel che allora era parso "nettamente differenziato" dai due Blocchi, aveva ora un'aria "assai più vaga e diluita" ${ }^{95}$. Il solito doppio discorso, insomma, per attizzare l'ardore nazionalista dei suoi e placare gli altrui timori. Pacifista o edulcorata, ad ogni modo, la Terza Posizione era lì, sotto gli occhi di tutti; a braccetto col destino manifesto argentino: il "rinascimento politico ed economico, sociale e culturale" dell'Argentina justicialista, infatti, le assegnava la conduzione della "crociata" di "solidarietà" foriera dell" "uomo nuovo d'America" e di nuove speranze per l'Europa in rovina. Terra eletta della "giustizia sociale", solo essa poteva indicare la via dell'armonia sopra gli estremismi capitalisti e totalitari.

Peron fu ridondante e autocelebrativo; enfatico al limite del tronfio. Tanto valeva fargli la tara, o accettarne la versione coniata per placare l'an-

90 AMRES, Buenos Aires a Madrid, 27 de mayo de 1947. Del rifiuto parlò Eva; non è detto fosse andata così; AMREC, Buenos Aires a Santiago, de julio de 1947.

91 AMRES, Roma a Madrid, 3 de julio de 1947.

92 Così confidò l'Ambasciatore D'Ormesson, véase AMREC, Santiago a Buenos Aires, de mayo de 1947.

93 Véase Zanatta, L.: "La reforma faltante...”, págs. 111-130; la versione di Eva in AMRES, Buenos Aires a Madrid, 19 de septiembre de 1947.

94 Perón, J. D.: Por la cooperación económica y la paz mundial, Ministerio de Relaciones Exteriores y Culto, Buenos Aires, 1947.

95 AMRES, Buenos Aires a Madrid, 7 de julio de 1947. 
sia statunitense, entrata poi nella vulgata: che Washington non temesse; la Terza Posizione era un'arma politica per i tempi di pace, ma in caso di guerra l'Argentina sarebbe stata al suo fianco ${ }^{96}$. Tutta retorica, allora? Per nulla; perché era proprio in tempo di pace che la Terza Posizione s'imponeva come un'espressa sfida politica e ideologica agli Stati Uniti. Peron non cessò di ripeterlo ad amici, alleati e simpatizzanti. Il suo discorso, disse, aveva avuto grande impatto in America Latina e in talune nazioni europee; seppur addolcita nei toni, la Terza Posizione avrebbe attratto molti paesi ansiosi di sottrarsi all' orbita dei due colossi ${ }^{97}$. In America Latina, confidò Peron, il suo governo stava conducendo "un'accorta manovra" per contrastare l'ingerenza statunitense ed ergersi a portavoce popolare; e i risultati cominciavano a vedersi. Per questo aveva seminato i suoi addetti operai nelle ambasciate. Washington l'accusava di spedire degli agitatori? Beh, non si sbagliava; era quel che voleva. Formare una coscienza comune tra i popoli ispanoamericani era possibile e dunque urgeva che l'Argentina, forte com'era, offrisse loro delle alternative economiche. Se poi la guerra fosse scoppiata, pensava, più che d'uno scontro tra i Blocchi avrebbe preso la forma di guerre civili nazionali dal cui esito sarebbe dipeso l'allineamento di un paese con l'uno o l'altro dei contendenti. A maggior ragione, dunque, s'imponevano justicialismo e Terza Posizione, i soli in grado di tenere dritta la barra anticomunista sanando gli abusi capitalisti che crescevano la malapianta.

Le parole di Peron, era prevedibile, fecero rumore e la polemica avvampò. L'Argentina, denunciò il Sunday Times, stava lavorando a un Blocco Latino; Franco n'era entusiasta e voleva coinvolgervi Salazar ${ }^{98}$. Lo spettro di quel Blocco aleggiava peraltro anche nelle cancellerie latinoamericane, sospettose dell'esuberanza argentina e turbate dal rischio d'inimicarsi Washington; a Rio e Santiago, per dire, non tardarono un istante a bocciarlo". In tanti gridarono "al lupo" e si scagliarono contro l'ombra dell'Asse Buenos Aires - Madrid che s'allungava sull'America Latina ${ }^{100}$; un'ombra che nel 1948 assunse i tratti castrensi del peruviano Odrìa e del

96 Van Der Karr, Perón y los Estados Unidos..., págs. 200-201.

97 AMRES, Buenos Aires a Madrid, 17 de julio de 1947.

98 Sunday Times, 3 de agosto de 1947.

99 Véase New York Times, 7 de agosto de 1947; AMREC, Buenos Aires a Santiago, 15 de septiembre de 1947.

100 Véase, per esempio, El Plata, Montevideo, 17 de enero de 1947; El Crisol, La Habana, 9 de junio de 1949. 
venezuelano Pérez Jìménez, entrambi cari a Peron ${ }^{101}$. Molti, insomma, intuivano l'enorme potenziale della Terza Posizione tra le masse dell'America Latina e ne temevano il contagio. Peron, capitava di leggere perfino sulla stampa brasiliana, era senz'altro autoritario, ma la sua Argentina era "ricca, prospera, dinamica e ambiziosa"; aveva resistito agli Stati Uniti ed eccola rispettata da tutti. E mentre essa stava "alla pari di Europa e Stati Uniti" il Brasile si accucciava all'ombra di Washington, senza nerbo né dignità ${ }^{12}$. Chi dubitava che l'Argentina stesse una spanna sopra gli altri e fosse votata a guidare il Sud America ${ }^{103}$ ? E perché non crederci, pensavano a Buenos Aires, quando tutti, in America Latina, perfino l'alta finanza, si lamentavano di Washington, tanto avara coi vicini quanto prodiga con l'Europa? ${ }^{104}$

Difatti Peron lanciò la sfida. Tutto, d'altronde, dalla crisi greca agli umori dei commilitoni statunitensi, ne rafforzava le convinzioni: la guerra era alle porte e molti paesi latini desideravano correre ai ripari temendo che una vittoria degli Stati Uniti li avrebbe consegnati alla loro mercè. Alla "nuova Argentina" sarebbe allora toccato di colmare il vuoto lasciato dalla scomparsa del comunismo ${ }^{105}$. Pensava ancora, Peron, che Truman vacillasse; quasi gli sfuggisse che gli Stati Uniti erano ormai determinati a condurre la lotta al comunismo e che ne pativa la sua ambizione di erigersene a campione brandendo la Terza Posizione ${ }^{106}$. Specie agli occhi dei paesi cattolici, dove Truman incoraggiava partiti e leader dalle intonse credenziali democratiche, come Adenauer, De Gasperi e Schuman, e disdegnava quelli, come Franco e Peron, dal pedigree più ingombrante; un po' per compiacere gli alleati e l'opinione pubblica e un po' per non dare armi alla propaganda moscovita. Peron, insomma, si cullò a lungo con l'idea che gli Stati Uniti tentennassero, che in America e in Europa ben pochi credessero in loro come difensori della cristianità e

101 Betancourt, R.: "La amenaza del totalitarismo derechista”, La Jornada, México, 3 de agosto de 1949; Peron e suoi prediligevano i governi militari, véase AMREP, Buenos Aires a Lima, 1 de diciembre de 1948.

102 O Jornal, 9 de enero de 1947, Jornal do Comercio, 22 de agosto de 1947.

103 AMNEP, Rio de Janeiro a Lisbona, 21 de noviembre de 1947.

104 AMREA, Rio de Janeiro a Buenos Aires, 19 de noviembre de 1947; Asunciòn a Buenos Aires, 17 de septiembre de 1947; sulla preferenza per l'Europa aveva da ridire anche l'Ambasciatore americano Bruce, véase AMRES, Buenos Aires a Madrid, 26 de agosto de 1947.

105 Così diceva Peron, véase AMRES, Buenos Aires a Madrid, 30 de septiembre de 1947.

106 Gli sfuggiva che se gli Stati Uniti si erano atteggiati a "mediatori" tra inglesi e sovietici, ormai avevano preso il posto dei primi contro i secondi, véase Crockatt, R.: The Fifty Years War, Routledge, London and New York, 1995. 
dunque che i venti di guerra fredda non tarpassero in alcun modo le ali alla Terza Posizione ${ }^{107}$.

Le cose, confidava Peron, volgevano al bello. In Brasile e Cile, dove debitamente aiutati crescevano i suoi fans, in Bolivia e Paraguay, saldamente sotto controllo, in Ecuador e chissà dove ${ }^{108}$. E d'altronde, se in patria era riuscito nell'impresa di convogliare fascisti e socialisti sulla sua terza via, perché non farcela altrove? Aspirante taumaturgo, ambiva a purgare l'Apra peruviana e i comunisti brasiliani d'incrostazioni marxiste e ubbie internazionaliste; bloccando, al contempo, la "mostruosa persecuzione" antifascista in Europa e accogliendone gli scampati nel suo caldo abbraccio. I popoli europei, pensava, non aspettavano altro ${ }^{109}$. L'Asse con la Spagna era vitale per misurare la sua capacità di ricomporre l'edificio smembrato della cattolicità; strappandola all'isolamento e riportandola per mano nella famiglia delle nazioni cattoliche, avrebbe sancito la leadership argentina sulle sponde latine dell'Atlantico e tracciato i contorni della Terza Posizione. Non a caso Peron "trasudava" più che mai "affetto" per la Spagna, "l'unico paese davvero anticomunista d'Europa", e la sua diplomazia lavorava a tutta, ovunque e in ogni modo per la causa spagnola ${ }^{110}$; purché fosse chiaro ch'egli, e nessun'altri, dirigeva le operazioni: non i portoghesi, che ci stavano timidamente provando ${ }^{111}$, né, soprattutto, gli Stati Uniti, ancora titubanti a sdoganare la Spagna in Europa ma assai meno in America $^{112}$. Le vostre azioni sono in continua crescita, comunicò Peron all' Ambasciata spagnola nell'ottobre 1947; "l' isolamento diplomatico della Spagna è finito", chiosò Bramuglia. A buon intenditore: se Franco vedeva luce in fondo al tunnel lo doveva a Peron, non certo a Truman, alla cui porta bussava invano da tempo ${ }^{113}$.

Comunque la si girasse, dunque, si trattasse di anticomunismo, Spagna o Americhe, di economia, difesa o sindacati, la Terza Posizione sfociava in competizione tra Argentina e Stati Uniti. Per "venderla", Peron

107 AMRES, Buenos Aires a Madrid, 30 de septiembre de 1947.

108 Ibidem.

109 AMRES, Buenos Aires a Madrid, 16 de enero de 1948. de 1947.

110 AMRES, Buenos Aires a Madrid, 30 de septiembre de , 9 de octubre de, 13 de noviembre

111 In Venezuela, ad esempio, véase Archivo General de Administraciòn, Madrid, AGDA, Caracas a Madrid, 29 de julio de 1947.

112 AMRES, Madrid a Buenos Aires, 5 y 8 de noviembre de 1948.

113 Franco chiese e ottenne molto da Peron, véase AMRES, Madrid a Buenos Aires, 17, 21, 28 de noviembre de 1947; ben poco, ancora, da Truman, véase Viñas, En las garras..., págs. 39-54. 
doveva mostrarsi così forte e capace da convincere i partner d'America e d'Europa ch'essa fosse più sicura e conveniente dell'alleanza con Washington per contenere il comunismo, promuovere sviluppo e giustizia sociale, proteggere la cristianità. Niente male, visti gli equilibri mondiali all'indomani della guerra. A misurarne le ambizioni arrivò allora il piano Marshall ${ }^{14}$. Per un verso Peron confidò che l'Argentina, a caccia di dollari per finanziare la sua ambiziosa politica industriale, ne avrebbe goduto; sperò, insomma, in massicci acquisti di grano e carne per i beneficiari del Piano, ma invano, vista l'ostilità di Washington verso le sue intenzioni politiche, la sua economia dirigista e i prezzi gonfiati pretesi dal ministro Miranda $^{115}$. Ma per un altro verso il piano Marshall fu un test per la Terza Posizione e un'occasione per magnificarne le virtù. Certo, i paesi latini che come Italia, Francia e Portogallo vi attinsero in quantità sotterrarono ogni eventuale interesse per le sirene peroniste; col piano Marshall, insomma, dileggiato da Miranda e schernito da Peron ${ }^{116}$, la Terza Posizione smarrì gran parte del suo orizzonte europeo e si "americanizzò" sempre più. Ma a quanti ne furono esclusi, come la Spagna, o lasciati ai margini, come l'America Latina, Peron pensò ch'essa avrebbe offerto un'alternativa. Eccolo allora coprire Franco di crediti a modo di "netta e perfino brutale replica" all'esclusione della Spagna dal piano Marshall ${ }^{117}$; spiegare a Washington che l'Argentina e il justicialismo, non i dollari statunitensi, avrebbero salvato l'America Latina da miseria e comunismo ${ }^{118}$; imprecare contro l'egoismo mercantilista statunitense e annunciare un piano ancor più utile ed efficace, un piano Peron perno di una nuova area del "peso" 119 . Per l'Ambasciatore argentino a Washington quel piano era già in atto e i vicini ne avrebbero tratto il maggior giovamento adottando la dottrina di Peron ${ }^{120}$; il senatore Molinari, solerte apostolo d'una zona d'influenza argentina, non

114 Dorn, G. J.: "Bruce Plan and Marshall Plan: The United States's Disguised Intervention against Peronism in Argentina, 1947-1950”, International History Review, a. 21, n. ${ }^{2}$ 2, 1999, págs. 331-351.

115 AMREC, Buenos Aires a Santiago, 12 de noviembre y 6 de diciembre de 1948; Peron rispose firmando accordi commerciali con alcuni paesi comunisti, véase AMRES, Buenos Aires a Madrid, 12 de junio de 1948; AMREP, Buenos Aires a Lima, 16 de junio de 1948.

116 AMRES, Buenos Aires a Madrid, 3 de abril de 1948; AMREC, Buenos Aires a Santiago, 6 de diciembre de 1948 .

117 Viñas, En las garras..., pág. 141; AMRES, Buenos Aires a Madrid, 30 de septiembre de 1947 y 3 de abril de 1948.

118 Van Der Karr, Perón y los Estados Unidos..., pág. 205.

119 AMAEI, Buenos Aires a Roma, 24 de junio y 23 de julio de 1948.

120 AMREA, Washington a Buenos Aires, 8 de octubre de 1947. 
si trattenne: il suo governo, disse, avrebbe aperto banche commerciali in vari punti dell'emisfero, compresi gli Stati Uniti, così bisognosi di "capitali, prodotti alimentari e materie prime" di cui l'Argentina abbondava ${ }^{121}$. Ma come immaginare, si chiedeva l'Ambasciatore italiano, che povera di mercato interno, industrie di base e fonti energetiche, in difficoltà coi suoi piani di sviluppo, l'Argentina sottraesse i paesi latini alla "soverchiante dipendenza" dagli anglosassoni" ${ }^{122}$ ?

\section{Di come Peron perse l'Europa e l'Argentina si americanizzò}

Peron restò fedele alla Terza Posizione, non ne modificò natura e confini; lo si è detto: occupava il cuore della sua Rivoluzione. A Bogotà, nell'aprile 1948, dove nacque l'Organizzazione degli Stati Americani, ebbe un'occasione ghiotta per ribadirne il senso; difatti l'Argentina combatté con ogni mezzo pur di impedire l'erosione, foss'anche minima, della sovranità dei singoli Stati. Non solo, ma intuendo che gli Stati Uniti, assorbiti dal piano Marshall, avrebbero disatteso le aspettative dei latini d'America, si lanciò contro la cooperazione multilaterale voluta da Washington per ottenere campo libero ai trattati bilaterali su cui aveva costruito la sua "tentacolare" influenza economica ${ }^{123}$. Peron amava chiamarla "politica sanmartiniana"; l'Argentina, pensava, ricca di risorse e capitali e forte dell'insofferenza dei vicini per la gravosa dipendenza dagli Stati Uniti, sarebbe stata il faro dell'integrazione economica ispanoamericana ${ }^{124}$. L'unione spirituale tra i paesi latini avrebbe così poggiato sulla necessaria comunione d'interessi. Forse, osservava Peron, gli anglosassoni non facevano altrettanto ${ }^{125}$

Lo stesso valeva per la lotta al comunismo. Truman aveva sperato che da Bogotà uscisse una dichiarazione anticomunista ed era noto che premeva sui governi americani perché rompessero con l'Unione Sovietica e i partiti comunisti. Ma Peron la pensava altrimenti e vi si oppose. Per i motivi noti: l'unica via efficace per annientare il comunismo, n'era certo, era quel-

121 "Dichiarazioni sorprendenti", notò l'Ambasciatore cileno, AMREC, Buenos Aires a Santiago, 26 de mayo de y 10 de julio de 1948.

122 AMAEI, Buenos Aires a Roma, 23 de julio de 1948.

123 AMRES, Buenos Aires a Madrid, 30 de marzo de 1948.

124 AMRES, Buenos Aires a Madrid, 8 de abril de 1948.

125 La Prensa, Buenos Aires, 1 de abril de 1948. 
la del suo justicialismo, del suo "Stato nuovo" che ne aveva troncato la crescita eliminando "lo sfruttamento capitalista" e migliorando il tenore di vita della popolazione ${ }^{126}$. Il suo anticomunismo, badava a dire, era a prova di bomba, ma non avrebbe aderito a "generici slogan anticomunisti e ideologici" solo "per soddisfare la politica estera degli Stati Uniti" e unirsi "al gregge" che accorreva al loro richiamo. Era lui, infatti, con la bandiera dell'indipendenza ispanoamericana salda tra le mani, l'argine più resistente al comunismo; colui, insomma, che impediva ai comunisti di vantare i meriti della lotta dei popoli latini per l'emancipazione dall'imperialismo. E ciò valeva nelle Americhe come nell'Europa latina, dove Peron non credeva che gli Stati Uniti avessero forza, determinazione e tatto sufficienti per arginare la marea comunista ${ }^{127}$. Il miraggio del Blocco Latino balenava ancora, dunque, tanto che nel maggio 1948, nonostante il trionfo della strategia statunitense nelle elezioni italiane, Bramuglia v'indugiava fantasticando uno scenario in cui la Spagna, sorretta da Peron, si sarebbe eretta a piattaforma della rinascita europea, pronta ad accogliere Francia e Italia, che pure con Franco non volevano avere a che fare ${ }^{128}$. Stessa cosa in America del Sud, dove lo spettro d'un Blocco con capitale a Buenos Aires non cessava d'inquietare Stati Uniti e Brasile, decisi a tutto per troncare le ambizioni di Peron, ch'erano certi avrebbe fatto un sol boccone dei piccoli e poveri paesi nati un tempo dal vicereame del Rio de la Plata ${ }^{129}$.

Ma non c'era nulla da temere, sbottò Peron ${ }^{130}$. La Terza Posizione, disse ai brasiliani, era la loro casa e le voci sulle minacciose mire argentine erano farina dell'"imperialismo capitalista", consapevole che uniti "formeremo un blocco solido e invincibile". "Organizziamoci, disse loro, come fanno gli slavi, i tedeschi, gli anglosassoni, per avere il nostro posto nel concerto mondiale". Come dimenticare "che siamo latini e che anche chi non lo è per sangue lo è per religione, tradizioni e costumi, poiché come noi ha ereditato la grande e sacra civiltà di Cristo tramandataci dalla latinità"? Come non scorgere nella Terza Posizione "il senso cristiano della giustizia sociale"? Il mondo era diviso? Certo, osservò Peron: tra due imperialismi, l'uno dedito allo sfruttamento dello Stato sull'uomo e l'altro

126 AMRES, Buenos Aires a Madrid, 3 de abril de 1948; La Prensa, 1 de abril de 1948.

127 AMRES, Buenos Aires a Madrid, 16 de enero de y 3 de abril de 1948.

128 AMRES, Buenos Aires a Madrid, 14 de mayo de 1948.

129 Di Blocchi regionali si discusse a Bogotà. Bramuglia ne riferì agli spagnoli, véase AMRES, Buenos Aires a Madrid, 3 de junio de 1948.

130 Folha Carioca, 10 de octubre de 1948; AMREC, Buenos Aires a Santiago, 11 de octubre de 1948. 
dell'uomo sull'uomo. In mezzo, brandendo Spada e Vangelo, troneggiava la Terza Posizione ${ }^{131}$. E a chi, come il diplomatico e deputato franchista Luca de Tena, gli chiedeva se quel "terzo blocco" animato da un "cristianesimo attivo" si sarebbe consolidato e tenuto estraneo all'orbita delle potenze, Peron rispondeva ch'era doveroso; e non solo nell'America ispanica, ma ovunque si desiderasse scacciare l'idra comunista, contro cui era pronto l'antidoto justicialista ${ }^{132}$.

Cattolicesimo, latinità, giustizia sociale, anticomunismo, antimperialismo erano gli ingredienti della visione peronista del mondo. Del loro impasto erano fatti justicialismo e Terza Posizione. Ma lungi dal correre verso la guerra che Peron dava per certa ${ }^{133}$ e che avrebbe rivelato ai popoli latini la saggezza e lungimiranza della Terza Posizione, il mondo s'assestava tra mille sobbalzi sulle trincee della guerra fredda ${ }^{134}$. L'acqua dove nuotare, insomma, le si prosciugava intorno mano a mano che cattolici e protestanti, latini e anglosassoni d'Europa e d'America si raccoglievano all'ombra dell'Occidente cristiano per fare fronte al comunismo ${ }^{135}$. E poi l'economia argentina era in affanno, vacche grasse non ce n'erano più $\mathrm{e}$ dollari in cassa neppure. Più che conveniente, la via di Washington cominciava a farsi obbligatoria: la Terza Posizione stava diventando una camicia di forza. Cominciò allora il lento, imbarazzato e zigzagante viaggio a Canossa di Peron, con la ratifica del trattato di difesa emisferica e l'assenso alla guerra in Corea; un viaggio costoso e causa di grosse falle nella coerenza e credibilità della Terza Posizione ${ }^{136}$. Perfino la politica economica si ammorbidì, tanto da corteggiare i capitali statunitensi e accedere al sospirato credito dall'Exim Bank; la Terza Posizione era innocua, garantì l'Ambasciatore statunitense ${ }^{137}$.

Peron, però, non l'aveva archiviata. Non poteva né voleva ${ }^{138}$. Cosa sarebbe rimasto, se no, della "nuova Argentina"? Come spiegarne l'abbandono ai descamisados, cresciuti a pane, Peron e odio antiyankee? Come

131 Véase AMREC, Buenos Aires a Santiago, de noviembre de 1948.

132 ABC, Madrid, 28 de noviembre de 1948.

133 Sarebbe scoppiata tra aprile e settembre del 1950, precisò, AMREC, Buenos Aires a Santiago, 3 de marzo de 1950.

134 Come constatò Bramuglia, véase AMRES, Buenos Aires a Madrid, 22 de enero de 1949.

135 Terza Posizione e Patto Atlantico facevano a pugni, osservò l'Ambasciatore cileno, véase AMREC, Buenos Aires a Santiago, 28 de mayo de 1949.

136 Come fu notato, véase AMREC, Buenos Aires a Santiago, 7 y 20 de julio de 1950.

137 AMREC, Washington a Santiago, 8 de abril de 1950; AMRES, Washington a Madrid, 28 de octubre de 1950.

138 AMRES, Washington a Madrid, 11 de mayo de 1950. 
ingranare la retromarcia e farsi posto sul predellino dell'Occidente mentre su Eva e Peron, santi della sovranità nazionale, montava un religioso culto della personalità? E poi la Terza Posizione viveva ormai di vita propria. La CGT stava addirittura muovendo alla conquista dei lavoratori latinoamericani, senz'altro ansiosi, per Peron, di sottrarsi alla tutela "comunista" di Lombardo Toledano e a quella "gialla" dell'AFL statunitense. A Washington, pensavano alla CGT, non capivano che "l'America iberica va capita com'è" e che s'era naturale che gli Stati Uniti guidassero i paesi anglosassoni, lo era anche che quelli latini ruotassero intorno all'Argentina $^{139}$. Anzi, non si capiva come dei paesi latini potessero aderire a una Confederazione sindacale americana di cui l'Argentina non era membro senza macchiarsi di "una vergognosa e umiliante" sottomissione "all'egemonia brutale e rapace di un potere imperialista".

Presa tra tanti fuochi, la Terza Posizione divenne allora un'assordante cacofonia; gli abbracci più caldi e gli insulti più violenti volarono da Buenos Aires verso Washington; venduti agli uni come pedaggi per saziare le masse peroniste e agli altri come furbe dissimulazioni che mai avrebbero distolto dalla retta via rivoluzionaria, o addirittura come magnanimi riconoscimenti di Peron al rinsavimento statunitense. Ma molto prima che il sole della Terza Posizione impallidisse sui cieli dell'America Latina, era già tramontato in Europa, talvolta ancor prima di sorgere. La sua "gamba" europea, infatti, fu perlopiù virtuale o zoppicante e destinata a crollare sotto gli scossoni della guerra fredda, che mentre erigeva un portentoso ponte sull'Atlantico Nord riduceva in polvere quello che la storia aveva innalzato, verso Sud, tra in popoli latini d'Europa e d'America.

Che ne fu del grande club dove Peron s'era illuso d'arruolare, insieme ai vicini latinoamericani e sotto il paterno sguardo pontificio, francesi e italiani, spagnoli e portoghesi? Sorvoliamo sulla Francia: non che l'Argentina le avesse negato cure e premure quando annaspava tra le macerie della guerra, ma l'idea d'un Blocco latino con capitale a Buenos Aires non l'attraeva per nulla. Per mille, comprensibili ragioni. Certo, a Parigi fioccavano i cultori d'una terza via tra i nascenti Blocchi, specie tra i socialisti, e taluni ne segnalarono perfino le affinità col disegno peronista ${ }^{140}$. Ma tra di essi e Peron, tra l'atmosfera francese pregna d'antifascismo e quella argentina che del fascismo portava lo stigma, ogni dì rinfocolato dagli atti d'amore per

139 Véase la lettera di F. Zapata, dirigente della CGT, a W. Green, Presidente della AFL, in AMREC, Buenos Aires, a Santiago, 27 de agosto de 1948.

140 AMREC, Buenos Aires a Santiago, 28 de mayo de 1949. 
Franco, nessuna intesa era pensabile. Lo si sapeva, ma nel dubbio lo ribadirono i fastidi francesi per la visita di Eva Peron, ricambiati dal suo sprezzo per l'aria parigina densa di "comunismo". L'idea che sottratta all'onta della disfatta e ammessa al tavolo dei vincitori, di nuovo in sella al suo grande impero e rigida nella grandeur, la Francia si stringesse a Franco sotto le mura vaticane per innaffiare la debole pianta della Terza Posizione peronista, superava la fantascienza. La Germania, la ricostruzione, le colonie: questi erano gli assilli francesi, i macigni che tenevano la Francia coi piedi ben saldi tra Europa e Nord America. La latinità le importava eccome, ma non alla corte di Peron e ancor meno se confusa con l'ispanità.

Quel ch'era vero per la Francia, a maggior ragione valeva per l'Italia sconfitta, occupata e in attesa che i vincitori ne stabilissero il destino. La guerra l'aveva spogliata di gran parte del capitale politico posseduto un tempo in America Latina e obbligata ad aggrapparsi al ponte sorto tra Europa e Nord America. La sua autonomia era minima, tanto più che le penose condizioni economiche e la delicata collocazione alla frontiera tra $\mathrm{i}$ Blocchi ne facevano una pedina fragile ma vitale della Guerra Fredda, decisamente poco propensa a imbarcarsi nell'avventura della Terza Posizione; specie se colma d'astio, come in Peron, per Washington. Certo, tra Italia e Argentina la storia aveva gettato un cavo d'acciaio che Peron fece il possibile per rinsaldare e De Gasperi non aveva alcun interesse a recidere. Dal cibo ai crediti agevolati, dai vantaggi commerciali al sostegno diplomatico, dall' accoglienza degli immigrati alle generose donazioni di Eva Peron, la "nuova Argentina" non lesinò sforzi per blandire e calamitare l'Italia. Finché, d'altronde, i piani statunitensi per l'Europa restarono incerti, parve godere dell'assenso vaticano, che peraltro coltivò accogliendo in fretta e appieno i reiterati inviti pontifici a soccorrere l'Italia. Privilegiò l'immigrazione italiana, per esempio, ripromettendosi di riservarla ai lavoratori cattolici accompagnati da un cospicuo numero di sacerdoti ${ }^{141}$. Il governo di Roma, pensava Peron, grato per tanto amore, avrebbe forse abbassato la guardia con Franco: limare le differenze, sanare le rotture, era indispensabile perché tra le nazioni latine e cattoliche affermassero la loro "personalità" dinanzi agli anglosassoni ${ }^{142}$.

141 AMRES, Santa Sede a Madrid, 13 de diciembre de 1946 y 31 de enero de 1947; AMRES, Roma a Madrid, 21 de febrero de 1947; la Commissione argentina sull'Emigrazione fu ricevuta e incoraggiata da Pio XII. Il padre Silva, salesiano vicino a Peron, ne assunse la direzione.

142 AMRES, Buenos Aires a Madrid, 17 de mayo de 1947; Bramuglia lo disse anche con l'Ambasciatore francese. 
Presto, però, Peron saggiò i limiti della sua influenza sulla Penisola e scrutò a fondo l'abisso scavato dalla guerra tra i destini dei due paesi; un conto, constatò, era rianimare il legame spirituale con l'Italia, tutt'altro arruolarla nella Terza Posizione. Lo rivelò la furia di Eva per la cortesia gelida del governo italiano ${ }^{143}$, specie di De Gasperi, peraltro reduce dal viaggio a Washington che ne aveva suggellato la scelta atlantica. Proprio De Gasperi, d'altronde, moderato, democratico, occidentalista, era l'antitesi di Peron, che al suo cospetto pareva l'epigone del vecchio cattolicesimo integralista, corporativo e ispanista; un epigone pronto, per di più, a prestare generosa ospitalità ai tanti fascisti in fuga e a favorirne la riorganizzazione in veste anticomunista, il che non gli giovava agli occhi del governo italiano, ansioso di liberarsi dell'ingombrante fantasma del fascismo e di non alimentare la propaganda comunista ${ }^{144}$. Come Truman, che nell'union sacrée dei popoli latini, Spagna franchista compresa, vedeva un'ombra minacciosa per il cattolicesimo democratico ${ }^{145}$. Stando dove stava, insomma, considerato che gli Stati Uniti l'avevano liberata e il peso della Santa Sede, si capiva che l'Italia avrebbe camminato sul binario tracciato da Truman e Pio XII; i quali, spinti dalla guerra fredda, dal 1947 si strinsero sempre più l'un l'altro, d'accordo, anche, sull'ammissione dell'Italia a piano Marshall e Patto atlantico, per cui il Vaticano si era discretamente speso $^{146}$.

Alla predica peronista della Terza Posizione restava dunque poco da fare in Italia. Tanto più che si sgonfiò da sé e talvolta gli sforzi di Peron per accattivarsi le simpatie italiane divennero perfidi boomerang. L'immigrazione, per dire, causò presto tensioni tra Roma e Buenos Aires e scontento in Vaticano ${ }^{147}$. Emersero, infatti, mille problemi: il governo argentino non aveva dato l'ospitalità garantita, i sindacati italiani fecero fuoco e fiamme contro i criteri confessionali, uno scandalo costrinse Peron a decapitare la Commissione per l'Immigrazione, e così via ${ }^{148}$. Non sorprende, dunque, la velata ironia sulla Terza Posizione dall'Ambasciatore

143 L'Italia le parve in decomposizione, véase AMRES, Buenos Aires a Madrid, 19 de septiembre de 1947.

144 Su Peron e gli esuli fascisti véase AMRES, Buenos Aires a Madrid, 16 de enero de 1948. 145 AMRES, Santa Sede a Madrid, 5 de mayo de 1948.

146 Véase Kirby, Religion and the Cold War...; AMRES, Ministerio de Asuntos Exteriores, Dirección de Santa Sede, 23 de marzo de 1949.

147 AMREA, Santa Sede a Buenos Aires, 16 de mayo de 1948, 30 de marzo de 1949.

148 AMREC, Buenos Aires a Santiago, 27 de junio de 1947, AMRES, Roma a Madrid, 3 de julio de 1947. 
d'Italia in Argentina ${ }^{149}$. Né che il governo italiano si premurasse di istruire i suoi rappresentanti che varcavano l'Atlantico: silenzio sulla Terza Posizione e niente lagne sugli Stati Uniti ${ }^{150}$.

Ma i frutti non colti a Parigi e Roma, non li dette neppure Lisbona. E dire che il corporativismo cattolico portoghese aveva sempre attratto Peron e i suoi, cui Salazar pareva un naturale candidato alla Terza Posizione; tanto più che covava sospetti sulle intenzioni statunitensi: non celavano, si chiedeva, un disegno imperiale minaccioso per le colonie africane del Portogallo ${ }^{151}$. Non solo; Salazar temeva anche che l'ondata demoliberale che spazzava l'Europa occidentale gli erodesse il regime e l'obbligasse a omologarsi. Non appena, però, gli fu offerto di salire sul treno precluso a Franco, lo colse al volo, togliendosi di dosso il tremendo peso d'un isolamento analogo a quello del vicino. D'altronde il suo Portogallo era più piccolo e innocuo, offriva preziosi vantaggi militari, vantava antichi legami con gli inglesi e la sua neutralità durante la guerra non recava, come nel caso spagnolo, l'infamante marchio del collaborazionismo con l'Asse. Certo, era una dittatura, e delle più dure, ma il suo arruolamento nel fronte anticomunista sollevava meno resistenze, sia negli Stati Uniti che in Europa. E poi c'era chi garantiva: Salazar stava lavorando bene e i portoghesi non se la passavano male, riferì il Nunzio apostolico a Lisbona all'inviato di Truman. Certo, capiva le critiche ai regimi iberici, ma la democrazia di tipo anglosassone, ricordava, era costata un lungo travaglio: pazienza, dunque, e soprattutto unità dell'Occidente cristiano contro il comunismo. Forse il Portogallo non aveva mostrato buona volontà accogliendo le richieste statunitensi d'impiantare delle basi militari alle Azzorre? ${ }^{152}$

Anche Salazar, dunque, interessato o meno alla Terza Posizione, si guardò bene dal sposarla e badò alla pelle del suo regime accomodandosi sullo sgabello riservatogli nel piano Marshall e nella Alleanza Atlantica; adeguandosi, cioè, ai dettami del bipolarismo. Non si può dire, d'altronde, che tra la Nueva Argentina e l'Estado Novo fosse scoppiata la passione. Anzi, l'intesa abbozzata a fine guerra si tramutò presto in diffidenza e delu-

149 AMREI, Buenos Aires a Roma, 23 de julio de 1948.

150 Albònico, A.: "Italia y Argentina, 1943-1955: política, emigración e información periodística", Estudios Interdisciplinarios de America Latina y el Caribe, v. 3, n. 1, enero-junio, 1992, págs. 41-58. pág. 89.

151 Nogueira, F.: Salazar. O Ataque (1945-1958), v. IV C.Ed. do Minho, Barcelos, 2000,

152 Di Nolfo, Vaticano e Stati..., págs. 554-557. 
sione. Per vari motivi. Intanto perché pur vantando una medesima matrice cattolica e corporativa a mezza via tra comunismo e liberalismo i due regimi scoprirono di non rassomigliarsi così tanto. Sia a Peron, uomo d'armi e d'industria, eletto e amato dagli operai, sia ad Eva, idolo dei descamisados, non piaceva granché quella grigia dittatura senza popolo, tutta incenso e campagne. Cos'aveva in comune con l'epica emancipazione peronista dei lavoratori? ${ }^{153}$ Ma neanche Salazar stravedeva per quel caudillo latinoamericano ricco e prepotente i cui addetti operai pretendevano di seminare il Vangelo justicialista nelle fabbriche portoghesi ${ }^{154}$. Cosa voleva? Esportare la Rivoluzione? Piantare le radici d'un nuovo, invadente Impero latino? ${ }^{955}$

Neppure l'uso disinvolto dell'arma alimentare era andato giù al governo portoghese. Tanto che se dapprima, viste le promesse argentine, aveva fatto orecchie da mercante agli sforzi dell'Emergency Economic Committee for Europe per indurre Peron a calmierare i prezzi del grano, in seguito li aveva condivisi. I prezzi esorbitanti a cui Miranda vendeva i cereali della Pampa per la Cancelleria di Lisbona erano "una vera speculazione" Certo, mirava a finanziarvi il piano quinquennale di Peron, ma così facendo seminava astio tra i partner, anche quelli latini, vanificando l'impegno con cui Bramuglia cercava di portare acqua al mulino della Terza Posizione ${ }^{157}$.

Il Portogallo, insomma, restò nel "mirino" di Peron, ma fu sempre più circospetto e si guardò dal fare passi senza concertarsi con la Spagna ${ }^{158}$. Di più: dato l'isolamento di Franco e l'inaffidabilità di Peron, Salazar non disdegnò l'idea di sfruttare le sue entrature presso gli Alleati per ritagliarsi un ruolo su misura, estraneo all'orizzonte d'un Blocco latino. Temeva, d'altronde, che l'Asse Madrid-Buenos Aires scalzasse quello, antico e solido, tra i cugini iberici. Perché, allora, non dimostrare a Franco che l'amicizia portoghese era un valido contrappeso alla dipendenza che per amore o per forza stava maturando nei confronti di Peron? ${ }^{159}$. Più che verso la Terza

153 AMRES, Buenos Aires a Madrid, 19 de septiembre de 1947.

154 AMNEP, Repartiçao dos Negocios Politicos, 11 de julio de 1952.

155 AMNEP, Madrid, Embajada de Argentina a Embaixada do Portugal, 9 de diciembre de 1947. Ai portoghesi, per dire, non piacque che l'Ambasciatore argentino a Madrid avocasse tutti i meriti di non avere abbandonato la Spagna.

156 AMNEP, Ministerio dos Negocios Estrangeiros. D.G. dos Negocios Economicos e Consulares, 5 agosto, 1946.

157 Bramuglia e Miranda, d'altronde, erano cane e gatto, véase AMRES, Buenos Aires a Madrid, 30 de julio de 1948.

158 AMNEP, Londra a Lisbona, 3 de agosto de 1947; AMAEA, Lisbona a Buenos Aires, 9 de agosto de 1948 .

159 Nogueira, Salazar..., pág. 108. 
Posizione, in sintesi, Peron e la guerra fredda spinsero Salazar nella più vasta trincea dell'Occidente cristiano e a competere con l'Argentina per accattivarsi la riconoscenza della Spagna ${ }^{160}$.

Verso il 1947, dunque, l'unico puntello europeo della Terza Posizione era la Spagna. Ma anch'esso traballava più di quanto si evincesse dalla magniloquente pompa dell'amicizia tra Franco e Peron; difatti crollò presto, lasciando una scia di malcelati rancori. Da allora l'Europa svanì dall'orizzonte di Peron. Bastò poco perché tra Madrid e Buenos Aires calasse la notte; appena pochi mesi perché evaporasse il ricordo dei fasti del viaggio di Eva in Spagna e del ministro Artajo a Buenos Aires; perché la grancassa montata alla firma del protocollo Franco - Peron scemasse in un sordo mugugno e Peron rinunciasse al sogno di fare esplodere "un grosso tuono sullo scacchiere mondiale" recandosi a Madrid ${ }^{161}$; perché il "piccolo Asse" latino si frantumasse e i suoi pezzi se ne andassero per strade diverse ${ }^{162}$.

C'erano problemi economici, va da sé; gli stessi per cui più d'uno giudicava la Terza Posizione una fatua chimera, un passo molto più lungo della gamba. "Purtroppo", scrisse il ministro degli Esteri argentino al suo pari spagnolo sul finire del 1949, non potremo più onorare i nostri accordi commerciali ${ }^{163}$. E non di lì a un anno, ma dall'indomani. Erano stati, ricordava, un generoso pegno del vincolo spirituale con la Madre Patria, ma ora le difficoltà finanziare obbligavano il governo di Peron a concentrarsi sul risanamento interno. Aveva un bel da dire, il ministro Paz, giovane e incolore meteora imposta da Eva allorché s'era liberata dell'odiato Bramuglia, che l'intesa tra i due paesi non ne avrebbe sofferto, legati com'erano da una "indissolubile comunione di ideali e di spirito". Era una pietosa foglia di fico sull'agonia di un'alleanza e sul tramonto del sogno peronista di una comunità di nazioni latine d'Europa e d'America. All'inizio del 1950 era ormai chiaro a tutti: tra Argentina e Spagna era sceso il gelo ${ }^{164}$.

Ma s'è vero che tra i due paesi pesava il contenzioso economico e che le crude cifre obbligavano l'Argentina a rivedere gli impegni, le cause del divorzio furono perlopiù politiche e ideologiche; lungi dall'esplodere di colpo, d'altronde, erano lievitate col tempo. Certo, Peron e Franco invoca-

160 AGDA, Caracas a Madrid, 9 de agosto de 1947.

161 AMRES, Buenos Aires a Madrid, 3 de abril de 1948.

162 Véase Rein, La salvación de..., págs. 187-204.

163 AMREA, Buenos Aires a Madrid, 27 de diciembre de 1949.

164 AMREC, Buenos Aires a Santiago, de enero de 1950. 
vano l'ispanità e la civiltà cattolica assediata dal comunismo, dalla secolarizzazione e dal bipolarismo tra una potenza atea e una protestante. Ma su natura, fini e confini della Terza Via cattolica avevano idee diverse; come sui rispettivi ruoli. Per Franco le frontiere della Terza Posizione erano troppo vaste, vaghe e pericolose. Come pensare, sbuffavano a Madrid, di includervi Francia e Italia, fautrice la prima d'una latinità ostile all'ispanità e ridotta la seconda a così poca cosa nelle Americhe da sconsigliare di ridarle il peso perso? Meglio, dunque, sorvolare sull'idea di un Blocco Latino, per di più inviso agli anglosassoni, e coltivarne uno ispanico, più utile e consono $^{165}$. Ma quel che a Franco serviva per rompere l'isolamento e proiettare la Spagna cattolica Oltreoceano era troppo timido e angusto per Peron: che non aveva antichi onori da custodire ma una nuova leadership da affermare; non un regime da difendere ma un'ideologia da esportare; non una potenza da placare, ma semmai da sfidare.

Tanti, è vero, nella chiassosa galassia peronista, celebravano il rincontro con la Madre Patria; ma non tutti. Cosa guadagnava la rivoluzione peronista legandosi al carro franchista, si chiedevano alcuni? Non era meglio superare il vecchio ispanismo spagnolo? Non avevano fatto altrettanto gli Stati Uniti con gli inglesi? L'ispanità è in auge e se ne fa un gran parlare, osservava Leonardo de Aldama, intellettuale, cattolico e peronista. Ma era davvero una "vitamina" benefica? Perché la Spagna, poi? L'Argentina non era forse una peculiare miscela di spagnoli e italiani, ricca di sangue indiano e d'influenze francesi? Non v'era ormai una vera e propria argentinità "nutrita di carburanti d'ogni dove"? Un'argentinità cattolica, integralmente cattolica, ma pregna di giustizia e carità, non della mistica spagnola dell" imperium catholicum" fatto "col sangue dei pagani trucidati"166. La sua voce, non a caso, era la "giustizia sociale" di Peron, così lontana dal soffocante clima repressivo, antipopolare e clericale che il padre Henàn Benìtez, confessore di Eva Peron e ideologo peronista, aveva respirato a Madrid. Tanto, a suo dire, che il popolo spagnolo sopportava Franco ma amava Peron ${ }^{167}$. E' che peronisti e franchisti si contendevano la guida dell'orbe ispanico e cattolico; dietro i sorrisi covava la rivalità. Seppur solo e bisognoso, Franco coltivava ambizioni americane ${ }^{168}$; all'Argentina doveva

165 AMRES, Madrid a Buenos Aires, 21 de mayo de 1947.

166 Aldama, L. de: La hispanidad como problema y destino, Universidad Nacional de Cuyo, Argentina, 1948.

167 AMRES, Buenos Aires a Madrid, 18 de junio de 1948; l'Ambasciatore spagnolo pensò bene di mettere sotto sorveglianza quell'uomo "sveglio, cattivo e senza scrupoli".

168 Delgado Gomez-Escalonilla, Diplomacia franquista... 
molto, ma non tanto da cedergli il testimone di leader dell'ispanità. Ben vengano le iniziative per la Spagna, in cui Peron era più che mai prodigo, avvertì Artajo nell'agosto 1947; che fosse chiaro, però, ch'essa non ne aveva responsabilità, né dava mandato a nessuno di agire in suo nome ${ }^{169}$. Libera e sovrana, insomma, non si sarebbe fatta condizionare dalla gratitudine. Anche a Peron, d'altronde, Franco portava gioia e dolori. Era un ponte per l'Europa, è vero, e ne accreditava la Terza Posizione nel mondo cattolico. Ma diveniva ingombrante al momento di corteggiare gli operai latinoamericani o di estendere l'influenza justicialista laddove Franco era tabù, come il Messico ${ }^{170}$.

Cattolici e corporativi, dunque, Franco e Peron erano divisi da tante cose; compresa la geografia: in caso di guerra, per dire, alla Spagna sarebbe stato difficile restare neutrale, assai più che all' Argentina ${ }^{171}$. Per non dire delle origini e basi dei loro regimi, sorto l'uno da una violenta reazione conservatrice e l'altro da una rivoluzione militare volta a prevenirne una sociale impugnandone le bandiere. E la guerra fredda? Franco vi sguazzava, vedendovi il compimento dei suoi neri presagi e la via d'uscita dall'isolamento, ma per Peron era la peste che avrebbe reso plumbeo il dominio statunitense in America Latina; e ancora: Franco si dimenava per tornare in gloria nel seno dell'Occidente, mentre Peron recitava ad arte e agitava come un vessillo la sua veste di pecora nera; e lo stesso con l'anticomunismo: per Franco era una patente di cittadinanza occidentale; per Peron il certificato della Terza Via justicialista.

Che tante differenze ne logorassero il matrimonio man mano che la guerra fredda scolpiva il globo e imponeva scelte era inevitabile. A misurarne la temperatura, d'altronde, c'era un termometro sensibilissimo: i rapporti con gli Stati Uniti. Ben presto, infatti, segnalò febbre, rivelando che ogni piccola fessura scavata dalla Spagna nella corazza statunitense apriva una falla nell' Asse con l'Argentina e ogni passo spagnolo verso l'alleanza occidentale assestava un colpo letale alla Terza Posizione. Non era un mistero. La Spagna, fu la caustica profezia del padre Benìtez a metà del 1948, non aspettava altro che consegnarsi mani e piedi agli Stati Uniti, anche al prezzo di scaricare la scomoda amicizia peronista ${ }^{172}$. Si capiva così l'inedita "freddezza" argentina per la causa spagnola, alle Nazioni Unite e

169 AMRES, Madrid a Buenos Aires, 20 de agosto de 1947.

170 AMREC, México a Santiago, 23 de febrero de 1949.

171 AMRES, Buenos Aires a Madrid, 16 de enero de 1948.

172 AMRES, Buenos Aires a Madrid, 18 de junio de 1948. 
altrove; tanto più che tra Washington e Madrid tirava aria nuova, la quarantena spagnola era colma di crepe e Franco galleggiava ormai anche senza il salvagente di Peron ${ }^{173}$. Proprio la Chiesa e i cattolici, d'altronde, cioè coloro nel cui nome Peron aveva issato la bandiera della Terza Posizione, guidavano la crociata per ricucire lo strappo tra Stati Uniti e Spagna; specie il cardinal Spellman e i suoi fedeli, in cui tanto sperava Franco per convincere Truman del ruolo chiave della Spagna "nella difesa della civiltà cristiana nell'Occidente europeo" 174 . Tante grazie all'America ispanica, dunque, così prodiga con la Madre Patria, disse Franco nel maggio 1949, ma la sua Spagna volgeva gli occhi anche a Washington ${ }^{175}$.

La Chiesa e la Santa Sede, si diceva: guide spirituali e mentori ideali, ad esse spettava tanta parte nel sorreggere l'impalcatura della Terza Posizione, nell'indicarvi la casa delle nazioni cattoliche in fuga dalla tirannia bipolare. Forse Peron non aveva voluto il Vaticano in cima ai destinatari del suo pomposo annuncio al mondo della Terza Posizione, pur sapendo di dispiacere a quanti, in America Latina, non lo amavano? ${ }^{176}$. Né perse occasione di vestire i panni dello statista devoto, impegnato a "dare orientamento cristiano e cattolico a tutta la vita nazionale" ${ }^{177}$; nei circoli diplomatici, difatti, nessuno dubitava della sintonia tra Peron e il Papa ${ }^{178}$. Finché il Vaticano prese pian panino a gettare acqua gelata sugli ardori terzisti del caudillo argentino. Ormai certo della sfida mortale del comunismo, infatti, e della determinazione di Truman a combatterlo brandendo la cristianità ancor più del liberalismo ${ }^{179}$, Pio XII si batté per convogliare i cattolici dietro gli Stati Uniti; tutti, compreso Franco, sul cui regime poteva storcersi il naso ma che a differenza di Peron chiedeva solo d'unirsi alla crociata anticomunista, senza contestarne guida, modi e fini ${ }^{180}$. La Santa Sede portò così acqua in quantità al mulino dell'Occidente, deviandola dallo stagnante canale della Terza Posizione. Anzi, la perseveranza argentina sulla Terza

173 AMRES, Buenos Aires a Madrid, 29 de noviembre de 1948.

174 AMRES, Santa Sede a Madrid, 28 de diciembre de 1948.

175 AMRES, Santa Sede a Madrid, 21 de mayo de 1949; Joaquìn Ruiz-Giménez, Ambasciatore spagnolo in Vaticano, sottolineò proprio questo passaggio.

176 AMNEP, México a Lisbona, 30 de julio de 1947.

177 Così riferì il padre Sepich, religioso argentino, al cardinal Tardini, Sostituto della Segreteria di Stato, AMREA, Santa Sede a Buenos Aires, 14 de julio de 1949.

178 AMREC, Buenos Aires a Santiago, 3 de junio de 1948.

179 Véase il carteggio Truman-Pio XII dell'estate 1948 in Di Nolfo, Vaticano e Stati..., págs. 582-585, 599-600.

180 Véase AMREC, Santa Sede a Santiago, 17 de febrero de 1948; Di Nolfo, Vaticano e Stati..., págs. 561-562, 617-618. 
Via prese a suonarle una forma sospetta e testarda di minare l'unità anticomunista. L'intesa tra Peron e Pio XII durò quindi lo spazio d'un mattino dando la stura ai sospetti e rancori sollevati a palate dall'imperterrito regalismo peronista.

Tant'è che sul finire del 1948, pietrificati i Blocchi intorno alla sorte di Berlino, tutto quel ch'era parso incensare Peron agli occhi vaticani aveva ormai preso un'altra luce: il grano ora abbondava e quello argentino era meno richiesto d'un tempo, visti anche i prezzi proibitivi fissati da Miranda; l'emigrazione verso il Rio de la Plata era stata inferiore alle attese e la sua gestione non era piaciuta granché in Vaticano ${ }^{181}$; Peron s'apprestava a conservare il Patronato nella nuova Costituzione, irritando il Papa e seminando dubbi su fini e fede del suo governo; neppure il cronico tira e molla con gli Stati Uniti aveva molti fans: causava danni e basta, osservava mons. Lombardi della Segreteria di Stato; tanto valeva rimediarvi ${ }^{182}$. E c'era altro: il crescente peso della Chiesa statunitense in Vaticano e in America Latina non suffragava certo la rigida distinzione peronista tra America cattolica e protestante, latina e anglosassone, impermeabili tra loro; l'assidua azione del cardinal Spellman, per dire, nutriva il panamericanismo a spese d'ispanità e latinità ${ }^{183}$.

Così, all'inizio del 1949, con Peron e Pio XII ai ferri corti per il Patronato $^{184}$, anche il nodo della Terza Posizione era ormai giunto al pettine: come conciliarne lo spirito neutrale e la vocazione antiamericana con gli sforzi della Santa Sede per accorpare le nazioni cattoliche all'alleanza occidentale? ${ }^{185}$ Tanto più che lo spettro comunista che ossessionava Pio XII pareva aleggiare anche in America Latina, al punto da indurre Truman a energiche contromisure e il Papa a felicitarsene con la Casa Bianca e con quei governi, come il cileno, determinati a rompere con Mosca e i comunisti locali ${ }^{186}$; all'opposto di Peron, certo invece che il justicialismo bastasse a sradicare il comunismo dall'orizzonte latinoamericano e che lo spauracchio sovietico servisse a placare la prepotenza di Washington.

181 AMREC, Santa Sede a Santiago, 10 de octubre de 1948; AMREA, Santa Sede a Buenos Aires, 30 de marzo de 1949.

182 AMREA, Santa Sede a Buenos Aires, 13 de octubre de 1948.

183 AMRES, Buenos Aires a Madrid, 13 de noviembre de 1947.

184 Zanatta, La reforma faltante...

185 La posizione di mons. Montini in AMRES, Dirección de Santa Sede, Informe para la Junta de Política Exterior, 23 de marzo de 1949.

186 Di Nolfo, Vaticano e Stati..., págs. 533-534; AMREC, Santa Sede a Santiago, 29 de diciembre de 1948. 
Difatti il malumore pontificio s'avvertì e tra la coppia presidenziale e il Nunzio Apostolico i rapporti andarono a catafascio. In Vaticano, per esempio, non erano piaciute le accuse di Peron agli Stati Uniti allorché nell'aprile 1948 era caduto assassinato Eliecer Gaitàn, il suo potenziale emule colombiano; tanto più che nei circoli occidentali se ne incolpava il "comunismo internazionale". Poco più tardi, poi, la Santa Sede non celò un certo scetticismo alla notizia dello sventato attentato a Peron ed Eva, tacque alle violente accuse rivolte a Washington dal Presidente argentino e partecipò con educata freddezza alla cerimonie inscenate dall'Ambasciata argentina per celebrare lo scampato pericolo, sprizzando rabbia per l'arresto di tre sacerdoti indiziati nel complotto e per lo scandalo ordito dalla stampa peronista ${ }^{187}$. E Pio XII si guardò bene, a fine anno, dal fare l'eccezione annunciata dall'Ambasciatore argentino sospendendo gli esercizi spirituali pur di accogliere il ministro Bramuglia ${ }^{188}$. Men che meno, infine, giovava la testardaggine con cui Peron assegnava a religiosi di provata fede peronista le più delicate ambasciate in Vaticano, dove suonavano conferma del suo inguaribile regalismo ${ }^{189}$.

Si capisce, dunque, che la Terza Posizione indisponesse la Santa Sede e che Pio XII le negasse il patrocinio morale su cui Peron aveva così contato. Il fatto era che la Terza Posizione - scrisse José Vasconcelos, l'intellettuale messicano ormai assurto a nume del cattolicesimo latinoamericano - nasceva nobile e cristallina dalla sacrosanta lotta di Peron con i potentati argentini; non solo, ma era "comprensibile", dato il disdegno per l'America Latina di quanti ne avevano avuto aiuto e simpatia; ma diveniva "inaccettabile" e "sospetta" di comunismo se predicava la neutralità nel mondo diviso in Blocchi. Come non chiedersi, allora, se l'Argentina avrebbe fatto contro i Soviet "il suo dovere al fianco della civiltà cattolica e occidentale"? ${ }^{190}$

\section{Epilogo}

Correvano già gli anni '50 quando la mistica della Terza Posizione, predicata dai potenti mezzi del peronismo, toccò lo zenit. Stampa, radio e

187 AMREC, Santa Sede a Santiago, 10 de octubre de 1948.

188 AMREC, Santa Sede a Santiago, 14 de diciembre de 1948. de 1949.

189 Il caso del padre Prato, mercedario, in AMREA, Santa Sede a Buenos Aires, 13 de agosto

190 Vaconcelos, J.: La Tercera Posición, El Siglo, Bogotá, 27 de julio de 1949. 
comizi, fiumi di soldi e parole intonarono a coro: di trionfo in trionfo il justicialismo si apriva strada tra i Blocchi, man mano che le sue virtù si rivelavano al mondo. In effetti non c'era aria di de profundis per la Terza Posizione, la quale, in fondo, esprimeva un astio verso il bipolarismo ben vivo a ogni latitudine. Tanto ch'essa ebbe ancora ritorni di fiamma e momenti di gloria, quando i successi di Vargas, Ibañez, Paz Estenssoro, Velazco Ibarra ed altri parvero per un istante ridarle fiato. Ma in realtà boccheggiava: perché l'economia argentina era in affanno e stentava a sostenerne i costi, perché gli Stati Uniti remavano contro e forte, perché molti governi dubitavano dell'altruismo di Peron, perché le Terze Posizioni erano tante quanto "i peronismi" e così via. Soprattutto, però, perché la guerra fredda le toglieva reclute e aria.

Cammin facendo, però, la Terza Posizione era cambiata. Certo, Peron invocava ancora l'unità dei popoli latini e cattolici del Vecchio e Nuovo Mondo, ma l'Europa era ormai persa: la guerra fredda aveva tranciato il cordone ombelicale tra la Terza Posizione e il suo humus europeo. Il nuovo e ferreo legame sorto allora a cavallo dell'Atlantico Nord per fronteggiare il comunismo avvolse in uno spesso involucro e cominciò a saldare nella più vasta idea d'Occidente cristiano quel che Peron e i suoi tempi avevano creduto insanabili fratture tra cattolici e protestanti, latini e anglosassoni. L'energia destinata a nutrire la pianta della Terza Posizione in Europa, insomma, servì in realtà a crescerne un'altra ben diversa. Dall'albero che aveva immaginato frondoso Peron vide così staccarsi poco a poco gli agognati frutti: Francia, Italia, Portogallo, perfino la Spagna; e il Papa, il più importante. Tutti maturati nell'orto del vicino, corsi al fianco degli Stati Uniti contro il comunismo.

Così, mentre covava sogni di gloria e cavalcava immensi scenari, mentre Bramuglia trionfava alle Nazioni Unite e Molinari vi candidava Eva Peron a grandi incarichi, mentre se ne vagheggiava un vertice con De Gasperi e De Gaulle o un tête à tête con Truman, Peron fu stritolato dall'implacabile ingranaggio della guerra fredda e l'Argentina ricondotta ai suoi confini naturali ${ }^{191}$. La Terza Posizione, allora, si americanizzò: smise i panni della civiltà cattolica sospesa su due continenti e vestì quelli del nazionalismo latinoamericano, ripose l'universalismo cattolico in nome del justicialismo argentino ${ }^{192}$, cestinò l'ispanismo e poggiò sulle più concrete

191 Véase AMREP, Buenos Aires a Lima, 23 de enero de 1948; AMREC, Santiago a Buenos Aires, 6 de diciembre de 1948; Van Der Karr, Perón y los Estados Unidos..., pág. 190.

192 La Nación, 20 de julio de 1950; AMREC, Buenos Aires a Santiago, 20 de julio de 1950. 
spalle del sindacalismo. Fino a perdersi tra mille contraddizioni, dilaniata tra gli imperativi economici che spingevano Peron sulla via di Washington e quelli ideologici che l'obbligavano a procedere per la strada ormai tracciata. Come abbandonare la Terza Posizione, infatti, senza rinnegare sé stesso, il proprio popolo, la propria storia? Il mondo solcato un tempo a grandi balzi dalla mente e dalla diplomazia di Peron gli si fece grande e l'Atlantico s'allargò finché la sponda europea sparì dalla sua vista. Odiati e amati, imitati e censurati ${ }^{193}$, gli Stati Uniti, la sua ossessione, restarono allora il suo unico orizzonte, il terminale obbligato della sua politica. Era l'ennesimo, lungo e doloroso passo del cammino latinoamericano dall'Europa alle Americhe. 